\title{
Submodular Optimization Problems and Greedy Strategies: A Survey
}

\author{
Yajing Liu ${ }^{1}$. Edwin K. P. Chong ${ }^{2}$. \\ Ali Pezeshki ${ }^{2}$. Zhenliang Zhang ${ }^{3}$
}

Received: date / Accepted: date

\begin{abstract}
The greedy strategy is an approximation algorithm to solve optimization problems arising in decision making with multiple actions. How good is the greedy strategy compared to the optimal solution? In this survey, we mainly consider two classes of optimization problems where the objective function is submodular. The first is set submodular optimization, which is to choose a set of actions to optimize a set submodular objective function, and the second is string submodular optimization, which is to choose an ordered set of actions to optimize a string submodular function. Our emphasis here is on performance bounds for the greedy strategy in submodular optimization problems. Specifically, we review performance bounds for the greedy strategy, more general and improved bounds in terms of curvature, performance bounds for the batched greedy strategy, and performance bounds for Nash equilibria.
\end{abstract}

Keywords Curvature · greedy strategy · Nash equilibrium · optimization · performance $\cdot$ submodular

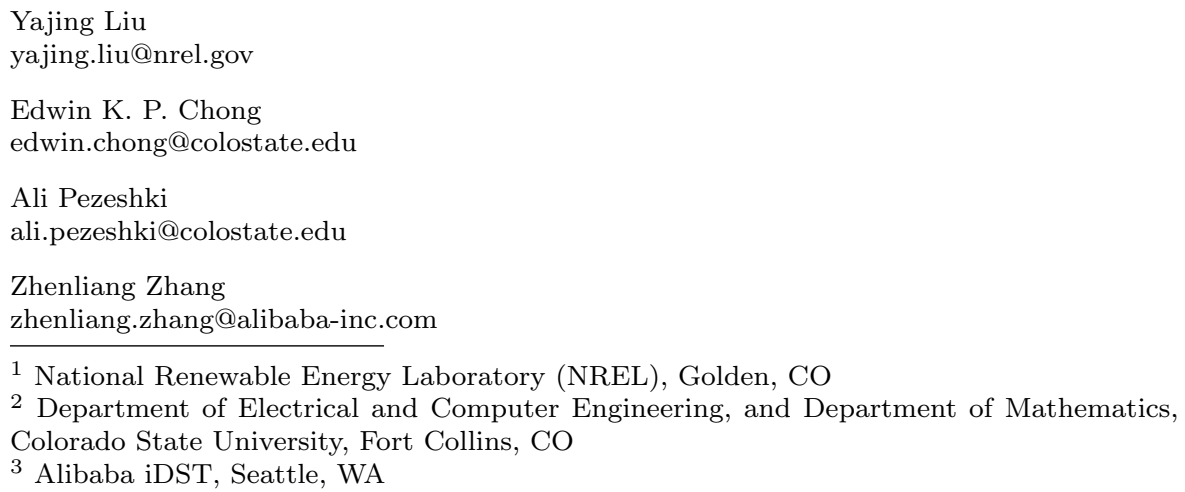




\section{Introduction}

We are often faced with choosing a set of actions from a ground set of actions to optimize an objective function. Such problems arise in a multitude of applications of interest to discrete-event dynamic system researchers. A specific example is the task assignment problem (Streeter and Golovin 2008 Zhang et al. 2016, Liu et al. 2018d), one of the fundamental combinatorial optimization problems in the study of optimization or operations research. This problem involves a number of agents and a number of tasks. Each agent successfully accomplishes a task with a certain probability and the aim is to assign the available tasks to a given number of agents such that the probability of accomplishing the tasks is maximized.

When the number of agents is relatively small, we can use brute-force search (Paar and Pelzl 2010) to enumerate all possible candidate solutions to find the optimal solution. However, when the number of agents is large, it is impractical to enumerate all the possible candidate solutions. At this point, we have to resort to approximation methods. One of the most well-studied approximation methods is the greedy strategy (Nemhauser et al. 1978), which starts with the empty set and iteratively adds to the current solution set an element that results in the largest gain in the objective function while satisfying the constraints. The greedy strategy yields an approximation to an optimal solution in a reasonable amount of time. The downside is that there is often no theoretical guarantee for the greedy strategy. But when the problem has a special property called submodularity, the greedy strategy is provably guaranteed to produce a solution with an objective value at least a constant scalar times the optimum value. Celebrated results by Fisher et al. (1978) and Nemhauser et al. (1978) prove that when the objective function $f$ is a monotone submodular set function with $f(\emptyset)=0$, the greedy strategy yields a $1 / 2$-approximation ${ }^{1}$ for a general matroid and a $\left(1-e^{-1}\right)$-approximation for a uniform matroid.

For set optimization problems, the objective function is not influenced by the order of actions. However, a great number of problems in engineering and applied science aim to optimally choose a string (finite sequence) of actions over a finite horizon to maximize an objective function whose value depends on the order of actions. The problem arises in sequential decision making in engineering, economics, management science, and medicine. A motivating example is the problem of scheduling sensors to detect targets (Li et al. 2009). Suppose that a given number of sensors are distributed in a sensor field to detect a certain number of targets. The goal is to activate sensors sequentially to maximize the total coverage area. If the coverage region of each sensor remains constant over time, the total coverage area is not influenced by the order of the sensors activated, and the problem becomes a set optimization problem. However, if the sensors are moving, then the total coverage area depends

1 The term $\beta$-approximation means that $f(G) / f(O) \geq \beta$, where $G$ and $O$ denote a greedy solution and an optimal solution, respectively. 
on the order of the sensors activated, which makes the problem fall into the framework of string optimization problems. The optimal solution to a string optimization problem is characterized by dynamic programming via Bellman's principle (Powell 2007). However, the approach suffers from the curse of dimensionality and is therefore impractical for many problems of interest. This motivates the study of approximation algorithms, among which the greedy strategy is easy to implement and has guaranteed performance bounds under certain conditions. For example, Streeter and Golovin (2008) prove that when the objective function is prefix and postfix monotone and has the diminishingreturn property (as defined later in the paper), the greedy strategy yields a $\left(1-e^{-1}\right)$-approximation.

In this paper, we review the performance guarantees for greedy strategies in submodular maximization problems. The paper is organized as follows. In Section 2, we review results that are related to choosing sets of actions. This involves introducing set functions, set optimization problem, performance bounds for the greedy strategy, examples, curvature, improved bounds, batched actions, and noncooperative games. In Section 3 , we review results related to choosing strings of actions. This involves introducing new notation and terminology, string optimization problem, performance of the greedy strategy, and applications. In Section 4 we conclude by listing a number of related papers that consider extensions and/or variation of greedy strategies and their performance bounds in combinatorial optimization problems.

\section{Sets of Actions}

In this section, we first introduce our notation for sets, properties of set functions, and set optimization problems. Then, we review various performance bounds for the greedy strategy.

\subsection{Set Functions}

Before we introduce functions defined on sets, we would like to introduce some similar and familiar properties for functions defined on real numbers. Consider a real function $f: \mathbb{R} \rightarrow \mathbb{R}$. The function $f$ is said to be monotone and submodular if it satisfies properties 1 and 2 below, respectively:

1. Monotone: $\forall x \leq y \in \mathbb{R}, f(x) \leq f(y)$.

2. Submodular: $\forall x \leq y \in \mathbb{R}, \forall z \in \mathbb{R}, f(x+z)-f(x) \geq f(y+z)-f(y)$

The 'monotone' property here simply means being 'nondecreasing'. The function in Fig. 1 satisfies the monotone property. From Fig. 1, we can see that the function is a concave function - adding $z$ to $x$ gains more than adding $z$ to $y$, which tells us that the additional value accrued by adding a number to a smaller number is larger than adding it to a bigger number. This is consistent with the inequality $f(x+z)-f(x) \geq f(y+z)-f(y)$ for $x \leq y$, so we say that 'submodularity' here boils down to 'concavity' in some sense. 


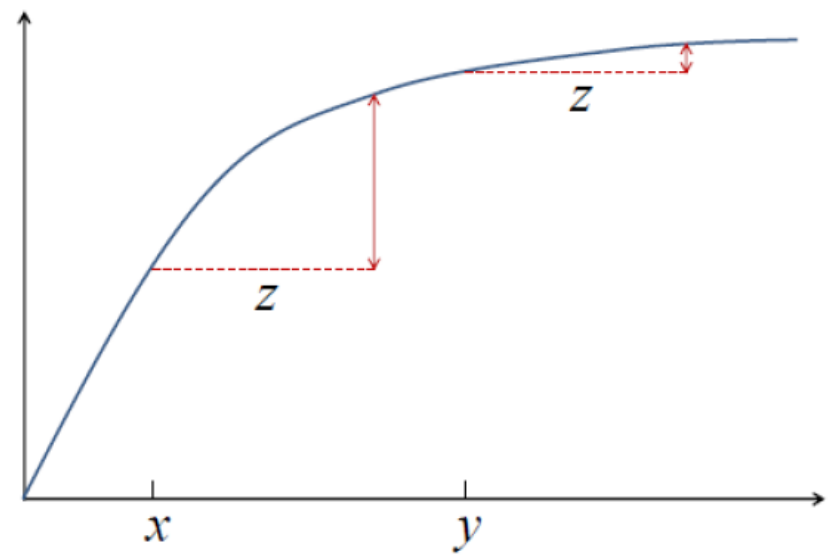

Fig. 1 Illustration of submodularity

In this paper, we want to go beyond the real line to a more general setting. Specifically, we will consider objective functions with multiple decision "actions" as arguments. The first setting is sets of actions, and the second one is strings (ordered sets) of actions. We introduce functions defined on sets first.

Let $X$ denote a ground set, which includes all possible actions. Let $2^{X}$ denote the power set of $X$, which includes all possible subsets of $X$. The size or cardinality of a set $S \in 2^{X}$ is denoted by $|S|$, and the empty set is denoted by $\emptyset$. Define a set function $f: 2^{X} \longrightarrow \mathbb{R}$. The set function $f$ is said to be monotone and submodular if it satisfies properties i and ii below, respectively:

i. Monotone: $\forall A \subseteq B \subseteq X, f(A) \leq f(B)$.

ii. Submodular: $\forall A \subseteq B \subseteq X$ and $\forall j \in X \backslash B, f(A \cup\{j\})-f(A) \geq f(B \cup$ $\{j\})-f(B)$.

Notice the similarity between these properties and those involving functions on the real line introduced earlier.

For convenience, we denote the incremental value of adding a set $T$ to the set $A \subseteq X$ as $\varrho_{T}(A)=f(A \cup T)-f(A)$ (following the notation in Conforti and Cornuéjols (1984)).

A set function $f: 2^{X} \longrightarrow \mathbb{R}$ is called a polymatroid set function (Boros et al. 2003) if it is monotone, submodular, and $f(\emptyset)=0$. Submodularity in property ii means that the additional value accruing from an extra action decreases as the size of the input set increases, and is also called the diminishing-return property in economics. Submodularity has many equivalent definitions; for example, $f: 2^{X} \longrightarrow \mathbb{R}$ is submodular if $\forall A, B \subseteq X$, $f(A)+f(B) \geq f(A \cup B)+f(A \cap B)$. For more equivalent definitions, see Nemhauser et al. (1978). 
The set function $f$ is called supermodular if $-f$ is submodular. Moreover, $f$ is called modular if it is both submodular and supermodular, i.e., for any $A \subseteq B \subseteq X$

$$
f(A)+f(B)=f(A \cup B)+f(A \cap B) .
$$

By induction, 11 implies that for any $S \subseteq X$,

$$
f(S)-f(\emptyset)=\sum_{s \in S}(f(\{s\})-f(\emptyset)) .
$$

By (2), $f-f(\emptyset)$ is additive when $f$ is modular. If $f(\emptyset)=0$, then $f(S)=$ $\sum_{s \in S} f(\{s\})$, which implies that $f$ is additive. It is also easy to check that $f$ is modular iff for any subset $S \subseteq X$,

$$
f(S)=\omega(\emptyset)+\sum_{s \in S} \omega(s)
$$

for some weight function $\omega: X \rightarrow \mathbb{R}$ (Krause and Golovin 2012).

There are many non-trivial examples of submodular or supermodular set functions. We only consider submodular maximization problems in this paper, so we only give submodular function examples. For supermodular examples, see Lovász (1983). To easily understand submodularity, we provide a simple example as follows.

Example 1 Sensor Coverage. Let $X$ be a family of locations in space where we can place sensors. If a sensor is placed at a particular location in space, it covers a circular area around it as illustrated in Fig. 2. Let $f(S)$ denote the total area covered if we place sensors at locations $S \subseteq X$ (see Fig. 2). The set function $f$ is submodular. An instance of submodularity is illustrated in the figure. As can be seen, the gain in adding sensor 3 after placing sensor 1 is larger than the gain in adding sensor 3 after placing sensors 1,2.

Submodular functions arise in many applications, such as the rank function of the matrix formed by its columns, weighted coverage functions, the rank function of a matroid, Shannon entropy, mutual information, cut capacity functions, some measurements on the graph, etc. (Lovász 1983; Krause and Golovin 2012

\subsection{Submodular Set Optimization Problem}

Submodular set optimization plays an important role in combinatorial optimization. It has a wide range of applications, including generalized assignment (Shmoys and Tardos 1993, Cohen et al. 2006, Nauss 2003, Fleischer et al. 2006, Bator |1957; |Korula et al.|2015; Vondrák |2008), matroid partition (Edmonds and Fulkerson |1965: Cunningham 1986, Knuth 1973), maximum cut (Goemans and Williamson 1995 | Sahni and Gonzalez |1976), maximum coverage location (Church and Velle 1974; Khuller et al. 1999: Cornuéjols et al. 1977), multi-agent coverage problem (Sun et al.|2017), leader-selection problem in 


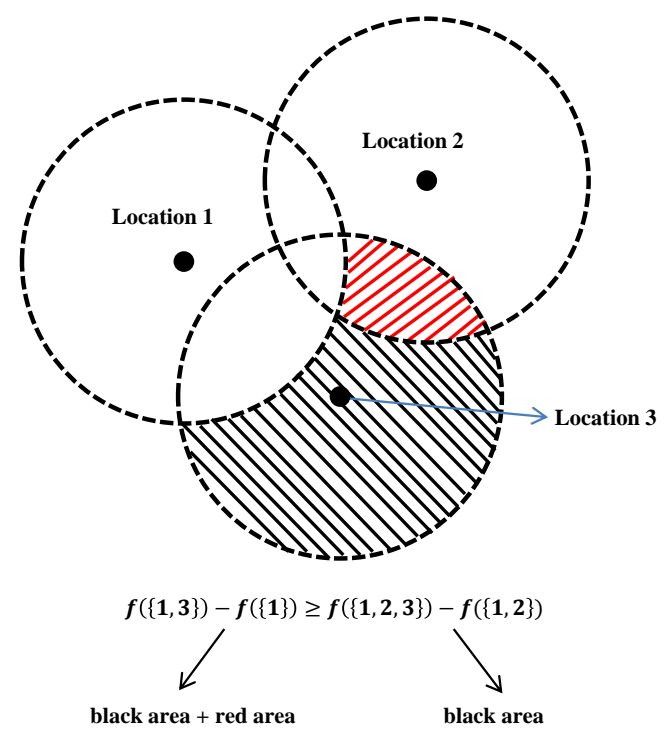

Fig. 2 Sensor coverage as an example of a submodular function

multi-agent systems (Clark and Poovendran 2011), welfare maximization (Korula et al. 2015, Vondrák 2008: Kapralov et al. 2013), and data summarization (Lin and Bilmes ||2011;| Badanidiyuru et al. ||2014|| Mirzasoleiman et al. 2017). The aim is to find a set of actions satisfying some constraints to maximize the objective function. The set optimization problem can be formulated as follows:

maximize $f(M), \quad$ subject to $M \in \mathcal{I}$,

where $\mathcal{I}$ is a non-empty collection of subsets of a finite set $X$, and $f$ is a real-valued submodular set function defined on the power set $2^{X}$ of $X$. Before proceeding any further with discussing optimization problem (4), we will need to introduce some concepts related to the constraint set $\mathcal{I}$.

Let $X$ be a finite set, and $\mathcal{I}$ be a non-empty collection of subsets of $X$. The collection $\mathcal{I}$ is said to be hereditary if it satisfies property i below and has the augmentation property if it satisfies property ii below:

i. Hereditary: For all $B \in \mathcal{I}$, any set $A \subseteq B$ is also in $\mathcal{I}$.

ii. Augmentation: For any $A, B \in \mathcal{I}$, if $|B|>|A|$, then there exists $j \in B \backslash A$ such that $A \cup\{j\} \in \mathcal{I}$.

The pair $(X, \mathcal{I})$ is called an independence system if it satisfies property i. In this case, the sets in $\mathcal{I}$ are called independent sets. A maximal independent set is an independent set that is not a subset of any other independent set (Conforti and Cornuéjols 1984). The independence system $(X, \mathcal{I})$ is called a matroid if it satisfies property ii $($ Edmonds 1970$)$. The pair $(X, \mathcal{I})$ is called a uniform matroid if $\mathcal{I}=\{S \subseteq X:|S| \leq K\}$ for a given $K$ (Nemhauser et al. 
1978). All maximal independent sets in a matroid have the same cardinality. We call this cardinality the rank of the matroid. In the uniform matroid above, the rank is $K$.

Example 2 We now give three example collections to illustrate the notions of independence systems and matriods. Let $X=\{a, b, c\}, \mathcal{I}_{1}=$ $\{\{a\},\{b\},\{a, c\},\{c\}, \emptyset\}, \mathcal{I}_{2}=\{\{a\},\{a, b\}\}$, and $\mathcal{I}_{3}=\{\emptyset,\{a\},\{b\},\{a, b\}\}$. It is easy to check that $\mathcal{I}_{1}$ satisfies the hereditary property but not augmentation, $\mathcal{I}_{2}$ satisfies augmentation but not the hereditary property, and $\mathcal{I}_{3}$ satisfies both hereditary and augmentation properties. Hence, $\left(X, \mathcal{I}_{1}\right)$ is an independence system, $\left(X, \mathcal{I}_{3}\right)$ is a matroid, and $\left(X, \mathcal{I}_{2}\right)$ is neither an independence system nor a matroid. The maximal independent sets in $\left(X, \mathcal{I}_{1}\right)$ are $\{b\}$ and $\{a, c\}$, and $\left(X, \mathcal{I}_{3}\right)$ only has one maximal independent set $\{a, b\}$.

Let $(X, \mathcal{I})$ be an independence system where $\mathcal{I}$ is nonempty, and let $S \subseteq X$ be an arbitrary subset of $X$. A basis of $S$ is a subset $B$ of $S$ that satisfies the following two conditions: 1 . It is an independent set; i.e., $B \in \mathcal{I}$. 2 . It is maximal; i.e., $B$ is not a subset of any other independent subset of $S$. The subset $B$ satisfying the above two conditions is also called a maximal independent subset of $S$. Define

$$
\begin{aligned}
& \text { lower rank of } S=\operatorname{lr}(S)=\min \{|B|: B \text { is a basis of } S\} \text {, } \\
& \text { upper rank of } S=\operatorname{ur}(S)=\max \{|B|: B \text { is a basis of } S\} \text {. }
\end{aligned}
$$

Note that $\operatorname{lr}(S)$ and $\operatorname{ur}(S)$ might not be well defined, depending on $S$. Note also that in the definition above, $S$ is not necessarily in $\mathcal{I}$. The number

$$
q(X, \mathcal{I})=\min \left\{\frac{\operatorname{lr}(S)}{\operatorname{ur}(S)}: S \subseteq X \text { and } \operatorname{ur}(S)>0\right\}
$$

is called the rank quotient of $(X, \mathcal{I})$ (Hausmann et al. 1980).

Example 3 To illustrate the concept of rank quotient, again consider the independence system $\left(X, \mathcal{I}_{1}\right)$ given in Example 2. We now consider all the subsets of $X$ and calculate their lower and upper ranks. If $S$ is a singleton (i.e., $\{a\},\{b\}$, or $\{c\})$, then $S$ has only one basis, which is $S$ itself. In this case, $\operatorname{lr}(S)=\operatorname{ur}(S)=1$, which means that $\operatorname{lr}(S) / \operatorname{ur}(S)=1$.

If $S=\{a, b\}$, its bases are $\{a\}$ and $\{b\}$. Again, $\operatorname{lr}(S)=\operatorname{ur}(S)=1$, which means that $\operatorname{lr}(S) / \operatorname{ur}(S)=1$. Note that $\{a, b\}$ is not a basis of $S$ because it does not belong to $\mathcal{I}_{1}$. If $S=\{a, c\}$, it has only one basis, which is itself, and again $\operatorname{lr}(S) / \operatorname{ur}(S)=1$. If $S=\{b, c\}$, its bases are $\{b\}$ and $\{c\}$, in which case $\operatorname{lr}(S) / \operatorname{ur}(S)=1$ again.

If $S=\{a, b, c\}=X$, the bases are $\{b\}$ and $\{a, c\}$. So, $\operatorname{lr}(S)=1$ and $\operatorname{ur}(S)=2$, which implies that $\operatorname{lr}(S) / \operatorname{ur}(S)=1 / 2$.

Because the rank quotient is the smallest among the ratios calculated above, we deduce that $q\left(X, \mathcal{I}_{1}\right)=1 / 2$. 
Example 4 As in Example 3, we can similarly check that $q\left(X, \mathcal{I}_{3}\right)=1$. In fact, the rank quotient of any matroid $(X, \mathcal{I})$ is equal to 1 , because for any susbset $S \subseteq X, \operatorname{lr}(S)=\operatorname{ur}(S)$ (Edmonds 1966). The rank quotient of an independence system $(X, \mathcal{I})$ can be regarded as a measure of how much $(X, \mathcal{I})$ differs from being a matroid.

For any independence system $(X, \mathcal{I})$, if there exist matroids $\left(X, \mathcal{I}^{i}\right)(1 \leq$ $i \leq p)$ such that $\mathcal{I}=\mathcal{I}^{1} \cap \cdots \cap \mathcal{I}^{p}$, then the pair $(X, \mathcal{I})$ is called the intersection of the matroids $\left(X, \mathcal{I}^{i}\right)$ (Hausmann et al. 1980).

Finding the optimal solution to (4) in general is NP-hard. The greedy strategy provides a tractable way to approximately solve the problem, which starts with the empty set, and incrementally adds an element to the current solution set giving the largest gain in the objective function under the constraints. Although the greedy strategy yields an approximate solution, its performance might be arbitrarily poor. However, when the optimization problem has the further special structure of being polymatroid, the greedy strategy has provable guarantees. The celebrated results by Fisher et al. (1978) and Nemhauser et al. (1978) show that the greedy strategy provides a good approximation to the optimal solution when the objective function is a polymatroid set function under both general matroid constraints and uniform matroid constraints. We will review the performance of the greedy strategy for (4) under different constraints in the following section.

\subsection{Performance Bounds for Greedy Strategy}

First we introduce definitions of the optimal strategy and the greedy strategy. Optimal Set: Any set $O$ is called an optimal solution of Problem (4) if

$$
O \in \underset{M \in \mathcal{I}}{\operatorname{argmax}} f(M),
$$

where argmax denotes the set of actions that maximize $f(\cdot)$.

\section{Greedy Algorithm:}

Input: A pair $(X, \mathcal{I})$, a set function $f: 2^{X} \rightarrow \mathbb{R}$

Output: A subset $G \in \mathcal{I}$

$G_{0} \leftarrow \emptyset$

For $i=1,2, \ldots$,

$g_{i} \leftarrow \underset{a \in X \backslash G_{-1}}{\operatorname{argmax}} f\left(G_{i-1} \cup a\right)$. If $g_{i} \neq \emptyset$, set $G_{i}=G_{i-1} \cup\left\{g_{i}\right\}$; otherwise, stop $a \in X \backslash G_{i-1}$

$G_{i-1} \cup\{a\} \in \mathcal{I}$

and set $G=G_{i-1}$.

Any output of the above algorithm is called a greedy solution. Note that there may exist more than one optimal solution or more than one greedy solution. How good is a greedy solution compared to an optimal solution in terms of the objective function? In the following theorems, we review performance bounds for the greedy strategy under different constraints. These bounds are worst-case performance bounds, which means that the greedy strategy performs much better than those bounds in many cases. 
Theorem 1 (Hausmann et al. 1980) Let $(X, \mathcal{I})$ be an independence system. If $f$ is additive on $X$, i.e., $f(S)=\sum_{s \in S} f(\{s\})$ for any subset $S \subseteq X$, then any greedy solution $G$ satisfies

$$
\frac{f(G)}{f(O)} \geq q(X, \mathcal{I})
$$

where $q(X, \mathcal{I})$ is the rank quotient defined in Section 2.2. Furthermore, for some function $f,(6)$ holds with equality.

Remark 1 When $(X, \mathcal{I})$ is a matroid, $q(X, \mathcal{I})=1$. By Theorem 1, the greedy strategy is optimal when $(X, \mathcal{I})$ is a matroid and the objective function is additive.

Remark 2 When $(X, \mathcal{I})$ is the intersection of $p$ matroids, then $q(X, \mathcal{I}) \geq 1 / p$ (Hausmann et al. 1980). So when $p=1$, i.e., $(X, \mathcal{I})$ is a matroid, the greedy strategy is optimal, which is consistent with Remark 1 .

Example 5 We provide an exampl $\phi^{2}$ to demonstrate the performance bound in Theorem 1. Let $X=\{s, t, u, v, w, x\}$, and consider the collection of subsets

$$
\begin{gathered}
\mathcal{I}=\{\emptyset,\{s\},\{t\},\{s, t\},\{u\},\{v\},\{w\},\{x\},\{u, v\},\{u, w\},\{u, x\},\{v, w\},\{v, x\}, \\
\{w, x\},\{u, v, w\},\{u, v, x\},\{u, w, x\},\{v, w, x\},\{u, v, w, x\}\} .
\end{gathered}
$$

Define a function $f$ such that $f(A)=\sum_{a \in A} f(\{a\})$. Let $f(\{s\})=$ 1.01, $f(\{u\})=f(\{v\})=f(\{w\})=f(\{x\})=1$, and $f(\{t\})=0$.

It is easy to check that $(X, \mathcal{I})$ is an independence system. If $S=$ $\{s, u, v, w, x\}$, it has bases $\{s\}$ and $\{u, v, w, x\}$, which results $\operatorname{in} \operatorname{lr}(S) / \operatorname{ur}(S)=$ $1 / 4$. Because the maximum cardinality of the maximal independent subsets of any $S \subseteq X$ is $4, \operatorname{lr}(S) / \operatorname{ur}(\mathrm{S}) \geq 1 / 4$ for any set $S \subseteq X$ with $\operatorname{ur}(S)>0$. Therefore, $q(X, \mathcal{I})=1 / 4$. The greedy solution is $G=\{s, t\}$ with $f(G)=1.01$ and the optimal solution is $O=\{u, v, w, x\}$ with $f(O)=4$, which satisfy the bound $f(G) / f(O) \geq q(X, \mathcal{I})$. In fact, the bound holds with equality if we lower $f(\{s\})$ to exactly 1 .

The following theorem bounds the performance of the greedy strategy when $(X, \mathcal{I})$ is the intersection of $p$ matroids and $f$ is a polymatroid set function.

Theorem 2 (Fisher et al. 1978) Let $(X, \mathcal{I})$ be the intersection of $p$ matroids and $f: 2^{X} \rightarrow \mathbb{R}$ a polymatroid set function. Then any greedy solution $G$ satisfies

$$
\frac{f(G)}{f(O)} \geq \frac{1}{1+p}
$$

Remark 3 The condition that $f$ is additive in Theorem 1 is stronger than the condition that $f$ is a polymatroid set function in Theorem 2 so the bound $1 / p$ in Theorem 1 is stronger than the bound $1 /(1+p)$ in Theorem 2 .

2 We thank the anonymous reviewer for this example. 
Remark 4 The bound $1 /(1+p)$ can be achieved for any positive integer $p$. When $p=1,(X, \mathcal{I})$ is a matroid, and the bound becomes $1 / 2$, which means that the greedy strategy yields $1 / 2$-approximation for general matroid constraints.

Remark 5 Theorem 2 requires that $f(\emptyset)=0$. If $f(\emptyset) \neq 0$, the following performance bound holds

$$
\frac{f(G)-f(\emptyset)}{f(O)-f(\emptyset)} \geq \frac{1}{1+p} .
$$

The following theorem provides a performance bound for the greedy strategy when $(X, \mathcal{I})$ is a uniform matroid and $f$ is a polymatroid set function.

Theorem 3 (Nemhauser et al. 1978) Let $(X, \mathcal{I})$ be a uniform matroid and $f: 2^{X} \rightarrow \mathbb{R}$ a polymatroid set function. Then any greedy solution $G_{K}$ satisfies

$$
\frac{f(G)}{f(O)} \geq 1-\left(1-\frac{1}{K}\right)^{K}>1-\frac{1}{e},
$$

where $K$ is the rank of the uniform matroid and $e$ is the base of the natural logarithm.

Remark 6 The bound $1-(1-1 / K)^{K}$ is stronger than the bound $1 /(1+p)$ when $p=1$ in Theorem 2 because the uniform matroid is a special matroid.

Remark 7 The bound $1-(1-1 / K)^{K}$ is decreasing in $K$ and tends to $1-$ $1 / e$ when $K$ goes to infinity. When $K=1$, the bound becomes 1 , which is consistent with the fact that the greedy strategy chooses the best action at each stage.

Remark 8 The bound $1-(1-1 / K)^{K}$ is tight, which means that it can be achieved for each $K$ (Nemhauser et al. 1978).

Remark 9 By Theorem 2 the greedy strategy only achieves a 1/2approximation under general matroid constraints. However, Calinescu et al. (2011) proved that a variant of the greedy strategy yields a $(1-1 / e)$ approximation under general matroid constraints.

\subsection{Examples}

We introduce two examples - a task scheduling problem and an adaptive sensing problem - to illustrate polymatroid set functions. In both problems, $(X, \mathcal{I})$ is a uniform matroid and hence the greedy strategy satisfies a $\left(1-e^{-1}\right)$ approximation.

Task Assignment Problem: The task scheduling problem was posed by Streeter and Golovin (2008), and was also analyzed in Zhang et al. (2016) and Liu et al. (2018d). In this problem, there are $n$ subtasks and a set $X$ of 
$N$ agents. At each stage, a subtask $i$ is assigned to an agent $a$, who accomplishes the task with probability $p_{i}(a)$. Let $X_{i}\left(\left\{a_{1}, a_{2}, \ldots, a_{k}\right\}\right)$ denote the Bernoulli random variable that signifies whether or not subtask $i$ has been accomplished after assigning the set of agents $\left\{a_{1}, a_{2}, \ldots, a_{k}\right\}$ over $k$ stages. Then $\frac{1}{n} \sum_{i=1}^{n} X_{i}\left(\left\{a_{1}, a_{2}, \ldots, a_{k}\right\}\right)$ is the fraction of subtasks accomplished after $k$ stages by employing agents $\left\{a_{1}, a_{2}, \ldots, a_{k}\right\}$. The objective function $f$ for this problem is the expected value of this fraction, which can be written as

$$
f\left(\left\{a_{1}, \ldots, a_{k}\right\}\right)=\frac{1}{n} \sum_{i=1}^{n}\left(1-\prod_{j=1}^{k}\left(1-p_{i}\left(a_{j}\right)\right)\right)
$$

The aim is to choose a set of agents to maximize this objective function.

Assume that $p_{i}(a)>0$ for any $a \in X$. Then it is easy to check that $f$ is monotone, submodular, and $f(\emptyset)=0$, which implies that $f$ is a polymatroid set function.

Adaptive Sensing: As our second example application, we consider the adaptive sensing design problem posed in Zhang et al. (2016) and Liu et al. (2018d). Consider a signal of interest $x \in \mathbb{R}^{2}$ with normal prior distribution $\mathcal{N}(0, I)$, where $I$ is the $2 \times 2$ identity matrix; our analysis easily generalizes to dimensions larger than 2 . Let $\mathbb{B}=\left\{\operatorname{Diag}(\sqrt{b}, \sqrt{1-b}): b \in\left\{b_{1}, \ldots, b_{N}\right\}\right\}$, where $b_{i} \in[0,1]$ for $1 \leq i \leq N$. At each stage $i$, we make a measurement $y_{i}$ of the form

$$
y_{i}=B_{i} x+w_{i}
$$

where $B_{i} \in \mathbb{B}$ and $w_{i}$ represents i.i.d. Gaussian measurement noise with mean zero and covariance $\sigma^{2} I$, independent of $x$.

The objective function $f$ for this problem is the information gain, which can be written as

$$
f\left(\left\{B_{1}, \ldots, B_{k}\right\}\right)=H_{0}-H_{k} .
$$

Here, $H_{0}=\frac{N}{2} \log (2 \pi e)$ is the entropy of the prior distribution of $x$ and $H_{k}$ is the entropy of the posterior distribution of $x$ given $\left\{y_{i}\right\}_{i=1}^{k}$; that is,

$$
H_{k}=\frac{1}{2} \log \operatorname{det}\left(P_{k}\right)+\frac{N}{2} \log (2 \pi e)
$$

where for $1 \leq j \leq k$

$$
P_{j}=\left(P_{j-1}^{-1}+\frac{1}{\sigma^{2}} B_{j}^{T} B_{j}\right)^{-1}
$$

is the posterior covariance of $x$ given $\left\{y_{i}\right\}_{i=1}^{j}$. The objective is to choose a set of measurements to maximize the information gain $f\left(\left\{B_{1}, \ldots, B_{K}\right\}\right)=H_{0}-H_{K}$.

It is easy to check that $f$ is monotone, submodular, and $f(\emptyset)=0$; i.e., $f$ is a polymatroid set function. 
2.5 Curvature

As we saw in Section 2.1, submodularity is a second-order property by analogy to concavity. If we can quantify this second order property, then we can get tighter bounds. One way to quantify the second order property is to use the total curvature, defined by Conforti and Cornuéjols (1984):

$$
c(f):=\max _{j \in X: \varrho_{j}(\emptyset) \neq 0}\left\{1-\frac{\varrho_{j}(X \backslash\{j\})}{\varrho_{j}(\emptyset)}\right\} .
$$

To see that this is a second-order property, rewrite it in terms of differences of differences:

$$
c(f):=\max _{j \in X: f(\{j\}) \neq f(\emptyset)}\left\{\frac{(f(\{j\})-f(\emptyset))-(f(X)-f(X \backslash\{j\}))}{f(\{j\})-f(\emptyset)}\right\} .
$$

For convenience, we use $c$ to denote $c(f)$ when there is no ambiguity. Note that $0 \leq c \leq 1$ when $f$ is a polymatroid set function, and $c=0$ when $f$ is modular. When $f$ is modular, $f-f(\emptyset)$ is additive. If we consider $f-f(\emptyset)$ as the objective function, then the greedy strategy achieves optimality. Therefore, in the rest of the paper, when we assume that $f$ is a polymatroid set function, we only consider $c \in(0,1]$.

Conforti and Cornuéjols (1984) provided performance bounds in terms of the total curvature for the greedy strategy under independence system, general matroid, and uniform matroid constraints, which will be reviewed as follows.

Theorem 4 (Conforti and Cornuéjols 1984) If $(X, \mathcal{I})$ is an independence system with $\operatorname{ur}(X)=K$ and $\operatorname{lr}(X)=k$, and $f$ is is a polymatroid set function with total curvature $c$, then any greedy solution $G_{K}$ satisfies

$$
\frac{f\left(G_{K}\right)}{f(O)} \geq \frac{1}{c}\left[1-\left(1-\frac{c}{K}\right)^{k}\right]
$$

and this bound is tight for all $0<c \leq 1$.

Theorem 5 Conforti and Cornuéjols 1984) If $(X, \mathcal{I})$ is a matroid and $f$ is a polymatroid set function with total curvature $c$, then any greedy solution $G_{K}$ satisfies

$$
\frac{f\left(G_{K}\right)}{f(O)} \geq \frac{1}{1+c}
$$

Moreover, if $(X, \mathcal{I})$ is a uniform matroid with rank $K$, then any greedy solution $G_{K}$ satisfies

$$
\frac{f\left(G_{K}\right)}{f(O)} \geq \frac{1}{c}\left[1-\left(1-\frac{c}{K}\right)^{K}\right]>\frac{1-e^{-c}}{c} .
$$

Remark 10 When $(X, \mathcal{I})$ is a matroid, the bound $1 /(1+c)$ is stronger than the bound $1 / 2$ in Theorem 2 because $c \in(0,1]$ when $f$ is a polymatroid set function and $1 /(1+c)$ is nonincreasing in $c$. 
Remark 11 The function $\left(1-e^{-c}\right) / c$ is nonincreasing in $c$, and therefore $(1-$ $\left.e^{-c}\right) / c \in\left[1-e^{-1}, 1\right)$ when $f$ is a polymatroid set function. Also it is easy to check that $\left(1-e^{-c}\right) / c \geq 1 /(1+c)$ for $c \in(0,1]$, which implies that the bound $\left(1-e^{-c}\right) / c$ for the uniform matroid constraints is stronger than the bound $1 /(1+c)$ for the general matroid constraints.

Remark 12 The two bounds in terms of the total curvature $c$ are both tight; for proofs, see Conforti and Cornuéjols (1984).

Remark 13 There are other notions of curvatures that can be used to characterize the second-order property of the set function $f$, such as the greedy curvature defined by Conforti and Cornuéjols (1984) and the elemental curvature defined by Wang et al. (2014). Performance bounds in terms of the corresponding curvatures were also derived by Conforti and Cornuéjols (1984) and Wang et al. (2014) under different constraints.

Example: Consider again the task assignment example from Section 2.4 For convenience, we only consider the special case $n=1$; our analysis can be generalized to any $n \geq 2$. For $n=1$, we have

$$
f\left(\left\{a_{1}, \ldots, a_{k}\right\}\right)=1-\prod_{j=1}^{k}\left(1-p\left(a_{j}\right)\right),
$$

where $p(\cdot)=p_{1}(\cdot)$.

Let us order the elements of $X$ as $a_{[1]}, a_{[2]}, \ldots, a_{[N]}$ such that

$$
0<p\left(a_{[1]}\right) \leq p\left(a_{[2]}\right) \leq \ldots \leq p\left(a_{[N]}\right) \leq 1 .
$$

Then by the definition of the total curvature $c$, we have

$$
c=\max _{j \in X}\left\{1-\frac{f(X)-f(X \backslash\{j\})}{f(\{j\})-f(\emptyset)}\right\}=1-\prod_{l=2}^{N}\left(1-p\left(a_{[l]}\right)\right)<1,
$$

which is consistent with our conclusion that $c \in[0,1]$.

\subsection{Improved Bounds}

The performance bounds of Conforti and Cornuéjols (1984) reviewed in Section 2.5. are the best bounds in terms of the total curvature $c$ for general matroid constraints and uniform matroid constraints, respectively. However, the total curvature $c$ depends on function values on sets outside the constraint matroid. If we are given a function defined only on the matroid, problem (4) still makes sense, but the bounds involving $c$ do not apply. Liu et al. (2018a, 2019 ) investigated modified bounds that overcome this drawback. The idea is first to extend a polymatroid set function defined on the matroid to one defined on the entire power set, and then apply the results from Conforti and 
Cornuéjols (1984). However, not every polymatroid function defined on the matroid can be extended to one defined on the entire power set.

In Liu et al. (2019), they first provide necessary and sufficient conditions for the existence of an incremental extension of a polymatroid set function defined on the uniform matroid of rank $k$ to one defined on the uniform matroid of rank $k+1$. Whenever a polymatroid objective function defined on a matroid can be extended to the entire power set, the greedy approximation bounds involving the total curvature of the extension apply. However, the bounds still depend on sets outside the matroid. Motivated by this, Liu et al. (2019) defined a new notion of curvature called partial curvature, involving only sets in the matroid. They derived necessary and sufficient conditions for an extension of the function to have a total curvature that is equal to the partial curvature. Moreover, they proved that the bounds in terms of the partial curvature are in general improved over the previous ones.

The following theorems state the necessary and sufficient conditions for the existence of an extension of a polymatroid set function defined on the uniform matroid of rank $k$ to one defined on the uniform matroid of rank $k+1$.

Theorem 6 (Liu et al. 2019) Let $f: \mathcal{I} \rightarrow \mathbb{R}$ be a polymatroid function defined on the uniform matroid of rank $k$. Then $f$ can be extended to a polymatroid function $g$ defined on the uniform matroid of rank $k+1$ if and only if for any $A \subseteq X$ with $|A|=k+1$, any $B \subset A$ with $|B|=k$, and any $a \in B$,

$$
f(B)-f(B \backslash\{a\}) \geq f\left(B^{*}\right)-f(A \backslash\{a\}),
$$

where $B^{*} \in \underset{B: B \subset A,|B|=k}{\operatorname{argmax}} f(B)$.

Construction: If $f$ is extendable, then an extension $g$ can be constructed as follows: For any $A$ with $|A| \leq k, g(A)=f(A)$; For any $A$ with $|A|=k+1$,

$$
g(A)=g\left(B^{*}\right)+d_{A},
$$

where $d_{A}$ satisfies

$$
0 \leq d_{A} \leq \min _{\substack{B: B \subset A,|B|=k \\ a: a \in B}}\left\{f(B)-f\left(B^{*}\right)+f(A \backslash\{a\})-f(B \backslash\{a\})\right\} .
$$

Note that $f: 2^{X} \rightarrow \mathbb{R}$ is itself an extension of $f$ from $\mathcal{I}$ to the entire $2^{X}$, and the extended $f: 2^{X} \rightarrow \mathbb{R}$ is a polymatroid function on $2^{X}$. Therefore, we have that $c(f) \geq d=\inf _{g \in \mathcal{E}_{f}} c(g)$, where $\mathcal{E}_{f}$ is the set of all polymatroid functions $g$ on $2^{X}$ that agree with $f$ on $\mathcal{I}$. So if a polymatroid set function defined on the matroid can be extended to one defined on the whole power set, applying the performance bounds in Theorem 5 results in the following theorem.

Theorem 7 (Liu et al. 2019) Let $(X, \mathcal{I})$ be a matroid of rank $K$ and $f: \mathcal{I} \rightarrow$ $\mathbb{R}$ a polymatroid function. If there exists an extension of $f$ to the entire power set, then any greedy solution $G_{K}$ to problem (4) satisfies

$$
\frac{f\left(G_{K}\right)}{f(O)} \geq \frac{1}{1+d}
$$


where $d=\inf _{g \in \mathcal{E}_{f}} c(g)$. In particular, when $(X, \mathcal{I})$ is a uniform matroid, any greedy solution $G_{K}$ to problem (4) satisfies

$$
\frac{f\left(G_{K}\right)}{f(O)} \geq \frac{1}{d}\left[1-\left(1-\frac{d}{K}\right)^{K}\right]>\frac{1}{d}\left(1-e^{-d}\right) .
$$

Remark 14 The bounds $1 /(1+d)$ and $\left(1-e^{-d}\right) / d$ apply to problems where the objective function is a polymatroid function defined only for sets in the matroid and can be extended to one defined on the entire power set. However, these bounds still depend on sets not in the matroid, because of the way $d$ is defined.

Then Liu et al. (2019) defined a new curvature called the partial curvature $b(h)$ as follows:

$$
b(h):=\max _{\substack{j, A: j \in A \in \mathcal{I} \\ h(\{j\}) \neq h(\emptyset)}}\left\{1-\frac{h(A)-h(A \backslash\{j\})}{h(\{j\})-h(\emptyset)}\right\}
$$

and the partial curvature satisfies that $b(f) \leq c(g)$ when $g$ is an extension of $f$ from $\mathcal{I}$ to $2^{X}$. The following theorem provides necessary and sufficient conditions for the existence of an extension $g$ to have $c(g)=b(f)$.

Theorem 8 (Liu et al. 2019) Let $(X, \mathcal{I})$ be a matroid and $f: \mathcal{I} \rightarrow \mathbb{R}$ a polymatroid function. Let $g: 2^{X} \rightarrow \mathbb{R}$ be a polymatroid function that agrees with $f$ on $\mathcal{I}$. Then $c(g)=b(f)$ if and only if

$$
g(X)-g(X \backslash\{a\}) \geq(1-b(f)) g(\{a\})
$$

for any $a \in X$, and equality holds for some $a \in X$.

Liu et al. (2019) provided the following improved bounds for the greedy strategy if there exists an extension $g$ of $f$ such that $c(g)=b(f)$.

Theorem 9 Liu et al. 2019) Let $(X, \mathcal{I})$ be a matroid of rank K. Let $g: 2^{X} \rightarrow$ $\mathbb{R}$ be a polymatroid function that agrees with $f$ on $\mathcal{I}$ such that $g(X)-g(X \backslash$ $\{a\}) \geq(1-b(f)) g(\{a\})$ for any $a \in X$ with equality holding for some $a \in X$. Then, any greedy solution $G_{K}$ to problem (4) satisfies

$$
\frac{f\left(G_{K}\right)}{f(O)} \geq \frac{1}{1+b(f)} .
$$

In particular, when $(X, \mathcal{I})$ is a uniform matroid, any greedy solution $G_{K}$ to problem (4) satisfies

$$
\frac{f\left(G_{K}\right)}{f(O)} \geq \frac{1}{b(f)}\left[1-\left(1-\frac{b(f)}{K}\right)^{K}\right]>\frac{1}{b(f)}\left(1-e^{-b(f)}\right)
$$


Remark 15 The bounds $1 /(1+b(f))$ and $\left(1-(1-b(f) / K)^{K}\right) / b(f)$ do not depend on sets outside the matroid, so they apply to problems where the objective function is only defined on the matroid, provided that an extension that satisfies the assumptions in Theorem 8 exists. When $f$ is defined on the entire power set, $b(f) \leq c(f)$, which implies that the bounds are stronger than those from Conforti and Cornuéjols (1984).

Next consider again the task assignment problem from Section 2.4. Liu et al. (2019) gave an extension $g$ of $f$ defined on the uniform matroid of rank 2 to the whole power set with $c(g)=b(f)$, which is reviewed as follows.

Example: Let $X=\left\{a_{1}, a_{2}, a_{3}, a_{4}\right\}, p\left(a_{1}\right)=0.4, p\left(a_{2}\right)=0.6, p\left(a_{3}\right)=0.8$, and $p\left(a_{4}\right)=0.9$. Then, $f(A)$ is defined as in $(9)$ for any $A=\left\{a_{i}, \ldots, a_{k}\right\} \subseteq X$. Let $K=2$, then $\mathcal{I}=\{S \subseteq X:|S| \leq 2\}$. It is easy to show that $f: \mathcal{I} \rightarrow \mathbb{R}$ is a polymatroid function.

The polymatroid function $g$ constructed using (11) while satisfying (12) and (16) from Liu et al. (2019) is of the following form:

$$
\begin{aligned}
& g\left(\left\{a_{1}, a_{2}, a_{3}\right\}\right)=f\left(\left\{a_{2}, a_{3}\right\}\right)+d_{\left\{a_{1}, a_{2}, a_{3}\right\}}=0.96 . \\
& g\left(\left\{a_{1}, a_{2}, a_{4}\right\}\right)=f\left(\left\{a_{2}, a_{4}\right\}\right)+d_{\left\{a_{1}, a_{2}, a_{4}\right\}}=1, \\
& g\left(\left\{a_{1}, a_{3}, a_{4}\right\}\right)=f\left(\left\{a_{3}, a_{4}\right\}\right)+d_{\left\{a_{1}, a_{3}, a_{4}\right\}}=1.02, \\
& g\left(\left\{a_{2}, a_{3}, a_{4}\right\}\right)=f\left(\left\{a_{3}, a_{4}\right\}\right)+d_{\left\{a_{2}, a_{3}, a_{4}\right\}}=1.04, \\
& g(X)=g\left(\left\{a_{2}, a_{3}, a_{4}\right\}\right)+d_{X}=1.08 .
\end{aligned}
$$

The total curvature $c$ of $g: 2^{X} \rightarrow \mathbb{R}$ is

$$
c\left(g_{2}\right)=\max _{a_{i} \in X}\left\{1-\frac{g(X)-g\left(X \backslash\left\{a_{i}\right\}\right)}{g\left(\left\{a_{i}\right\}\right)-g(\emptyset)}\right\}=0.9=b(f)<c(f)=0.992 .
$$

By Theorem 9, the greedy strategy for the task scheduling problem satisfies the bound $\left(1-(1-b(f) / 2)^{2}\right) / b(f)=0.775$, which is better than the previous bound $\left(1-(1-c(f) / 2)^{2}\right) / c(f)=0.752$.

\subsection{Batch Actions}

Suppose we batch the selected actions into batches of size $k$. What results is the $k$-batch greedy strategy, which starts with the empty set and iteratively adds to the current solution set a batch of elements with the largest gain in the objective function under the constraints. The greedy strategy we considered in Sections 2.3 2.5 is a special case of the batched greedy with batch size equal to 1 . Intuitively, larger $k$ should result in better performance, albeit at the expense of increasing computational complexity. But how do the previous bounds improve as a function of $k$ ? In this section, we review performance bounds for the $k$-batch greedy strategy.

We start by introducing the $k$-batch greedy strategy as follows. Consider again problem (4) and write the maximal cardinality of the sets in $\mathcal{I}$ as $K=$ $k(l-1)+m$, where $l, m$ are nonnegative integers and $0<m \leq k$. Note that $m$ is not necessarily the remainder of $K / k$, because $m$ could be equal to $k$. 
This happens when $k$ divides $K$. The $k$-batch greedy strategy is as follows (Liu et al. 2018c d):

Step 1: Let $S^{0}=\emptyset$ and $t=0$.

Step 2: Select $J_{t+1} \subseteq X \backslash S^{t}$ such that $\left|J_{t+1}\right|=k, S^{t} \cup J_{t+1} \in \mathcal{I}$, and

$$
f\left(S^{t} \cup J_{t+1}\right)=\max _{J \subseteq X \backslash S^{t} \text { and }|J|=k} f\left(S^{t} \cup J\right) ;
$$

then set $S^{t+1}=S^{t} \cup J_{t+1}$.

Step 3: If $t+1<l-1$, set $t=t+1$, and repeat Step 2 .

Step 4: If $t+1=l-1$, select $J_{l} \subseteq X \backslash S^{l-1}$ such that $\left|J_{l}\right|=m, S^{l-1} \cup J_{l} \in \mathcal{I}$, and

$$
f\left(S^{l-1} \cup J_{l}\right)=\max _{J \subseteq X \backslash S^{l-1} \text { and }|J|=m} f\left(S^{l-1} \cup J\right) .
$$

Step 5: Return the set $S=S^{l-1} \cup J_{l}$ and terminate.

Any set generated by the above procedure is called a $k$-batch greedy solution. For the above strategy, there are $l$ steps in total, and exactly $k$ actions are selected at each of the first $l-1$ steps but the final step may select fewer than $k$ actions. A similar batched greedy strategy is investigated by Hausmann et al. (1980) called the $(\leq k)$-greedy strategy, where at most $k$ actions are selected at each stage.

The performance of the $k$-batch greedy strategy under uniform matroid constraints was first investigated by Nemhauser et al. (1978), stated as follows.

Theorem 10 (Nemhauser et al. 1978) If $(X, \mathcal{I})$ is a uniform matroid of rank $K$ and $f$ is a polymatroid set function, then any $k$-batch greedy solution $S$ satisfies

$$
\frac{f(S)}{f(O)} \geq 1-\left(1-\frac{m}{k l}\right)\left(1-\frac{1}{l}\right)^{l-1} .
$$

Remark 16 When $m=k$, i.e., the batch size $k$ divides the rank $K$, the bound is tight; see Nemhauser et al. (1978) for proof.

By introducing the total $k$-batch curvature

$$
c_{k}:=\max _{I \in \hat{X}}\left\{1-\frac{\varrho_{I}(X \backslash I)}{\varrho_{I}(\emptyset)}\right\},
$$

where $\hat{X}=\left\{I \subseteq X: \varrho_{I}(\emptyset) \neq 0\right.$ and $\left.|I|=k\right\}$, Liu et al. (2018d) derived performance bounds in terms of $c_{k}$ for the $k$-batch greedy strategy under both general matroid and uniform matroid constraints, and investigated the monotoneity of the performance bounds with respect to the batch size $k$.

Theorem 11 (Liu et al. $2018 d)$ Assume that $f$ is a polymatroid set function. When $(X, \mathcal{I})$ is a general matroid, then any $k$-batch greedy solution satisfies

$$
\frac{f(S)}{f(O)} \geq \frac{1}{1+c_{k}}
$$

When $(X, \mathcal{I})$ is a uniform matroid, then any $k$-batch greedy solution satisfies

$$
\frac{f(S)}{f(O)} \geq \frac{1}{c_{k}}\left[1-\left(1-\frac{c_{k}}{l} \frac{m}{k}\right)\left(1-\frac{c_{k}}{l}\right)^{l-1}\right] .
$$


Remark 17 When $k=1$, the bound for general matroid constraint becomes $1 /(1+c)$ and the bound for uniform matroid constraints becomes $(1-(1-$ $\left.c / K)^{K}\right) / c$, which is consistent with the results in Theorem 5 .

Remark 18 The total $k$-batch curvature is nonincreasing in $k$, i.e., $c_{k_{2}} \leq c_{k_{1}}$ whenever $k_{2} \geq k_{1}$ (Liu et al. 2018d).

Remark 19 Based on Remark 18, we can discuss the monotoneity of the bounds for both general matroid and uniform matroid constraints. The bound $1 /\left(1+c_{k}\right)$ for general matroid constraints is monotone in $k$. For uniform matroid constraints, when the batch size $k$ divides $K$, the bound becomes

$$
\frac{1}{c_{k}}\left[1-\left(1-\frac{c_{k}}{l}\right)^{l}\right]
$$

which is monotone in $k$. Moreover,

$$
\frac{1}{c_{k}}\left[1-\left(1-\frac{c_{k}}{l}\right)^{l}\right]>\frac{1-e^{-c_{k}}}{c_{k}} \geq \frac{1}{1+c_{k}},
$$

which means that the bound for uniform matroid constraints is better than the bound for general matroid constraints. However, if $k$ does not divide $K$, the exponential bound might be worse than the harmonic bound. For example, when $K=100, k=80$, and $c_{k}=0.6$, the exponential bound is 0.5875 , which is worse than the harmonic bound 0.6250 (Liu et al. 2018d).

Examples: Now consider again the task assignment and adaptive sensing problems from Section 2.4 to demonstrate that the total curvature $c_{k}$ decreases in $k$ and the performance bound for a uniform matroid increases in $k$ under the condition that the batch size $k$ divides the rank $K$

Task Assignment Problem: We still order the elements of $X$ as $a_{[1]}, a_{[2]}, \ldots, a_{[N]}$ such that

$$
0<p\left(a_{[1]}\right) \leq p\left(a_{[2]}\right) \leq \ldots \leq p\left(a_{[N]}\right) \leq 1 .
$$

Then by the definition of the total curvature $c_{k}$, we have

$$
c_{k}=\max _{i_{1}, \ldots, i_{k} \in X}\left\{1-\frac{f(X)-f\left(X \backslash\left\{i_{1}, \ldots, i_{k}\right\}\right)}{f\left(\left\{i_{1}, \ldots, i_{k}\right\}\right)-f(\emptyset)}\right\}=1-\prod_{l=k+1}^{N}\left(1-p\left(a_{[l]}\right)\right) .
$$

From the expression of $c_{k}$, we can see that $c_{k}$ is nonincreasing in $k$, but when $N$ is large, $c_{k}$ is close to 1 for each $k$.

To numerically evaluate the relevant quantities here, Liu et al. (2018d) randomly generated a set $\left\{p\left(a_{i}\right)\right\}_{i=1}^{30}$. In Fig. 3, they considered $K=20$, and batch sizes $k=1,2, \ldots, 10$. Fig. 3 shows that the exponential bound for $k=3,6,8,9$ is worse than that for $k=1,2$, which illustrates our earlier remark that the exponential bound for the uniform matroid case is not necessarily monotone in $k$ even though $c_{k}$ is monotone in $k$. Fig. 3 also shows that the exponential bound $\left(1-\left(1-c_{k} / l \cdot m / k\right)\left(1-c_{k} / l\right)^{l-1} / c_{k}\right.$ coincides with $(1-(1-$ 


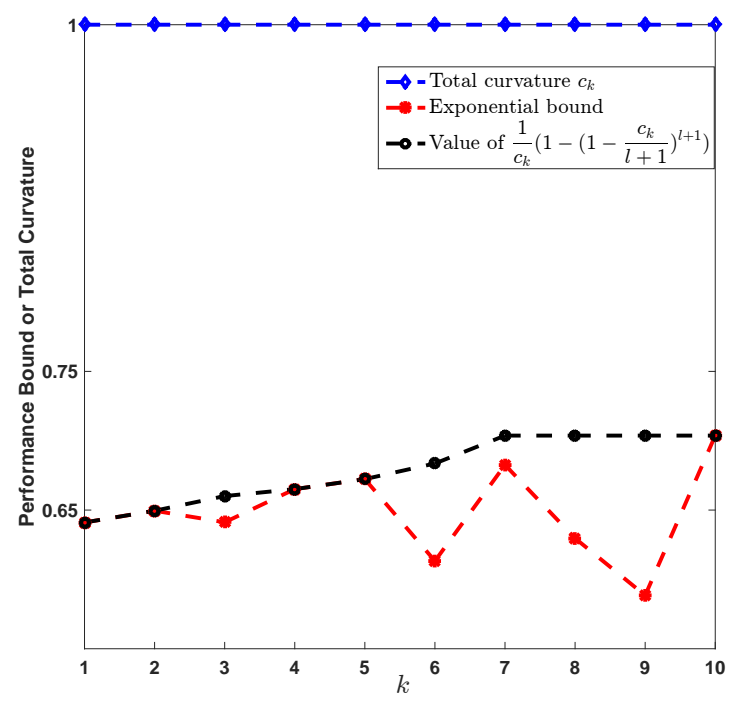

Fig. 3 Total curvature/performance bounds for greedy strategy in task assignment problem

$\left.\left.c_{k} / l\right)^{l}\right) / c_{k}$ for $k=1,2,4,5,10$ and it is nondecreasing in $k$, which illustrates our remark that the exponential bound is nondecreasing in $k$ under the condition that $k$ divides $K$. Owing to the nature of the total curvature for this example, it is not easy to see that $c_{k}$ is nonincreasing in $k$ (all $c_{k}$ values here are very close to 1).

Adaptive Sensing: For convenience, set $\sigma=1$. Then, we have

$$
\begin{aligned}
c_{k} & =\max _{J_{k} \subseteq X,\left|J_{k}\right|=k}\left\{1-\frac{f(X)-f\left(X \backslash J_{k}\right)}{f\left(J_{k}\right)}\right\} \\
& =\max _{J_{k} \subseteq X,\left|J_{k}\right|=k}\left\{1-\frac{\log (s t)-\log \left(s-\sum_{i: e_{i} \in J_{k}} e_{i}\right)\left(t-\sum_{i: e_{i} \in J_{k}}\left(1-e_{i}\right)\right)}{\log \left(1+\sum_{i: e_{i} \in J_{k}} e_{i}\right)\left(1+\sum_{i: e_{i} \in J_{k}}\left(1-e_{i}\right)\right)}\right\},
\end{aligned}
$$

where $X=\left\{B_{1}, \ldots, B_{N}\right\}, s=1+\sum_{i=1}^{N} e_{i}$, and $t=1+\sum_{i=1}^{N}\left(1-e_{i}\right)$.

We already saw that the exponential bound for the uniform matroid case is not necessarily monotone in $k$ from the task assignment problem, so we will only consider the case when the batch size $k$ divides $K$. Liu et al. (2018d) considered $K=24$ for $k=1,2,3,4,6,8$ in Fig. 4 . The figure shows that the curvature decreases in $k$ and the exponential bound increases in $k$ since $k$ divides $K$ for $k=1,2,3,4,6,8$, which again demonstrates the claim that $c_{k}$ decreases in $k$ and the exponential bound increases in $k$ under the condition that $k$ divides $K$. 


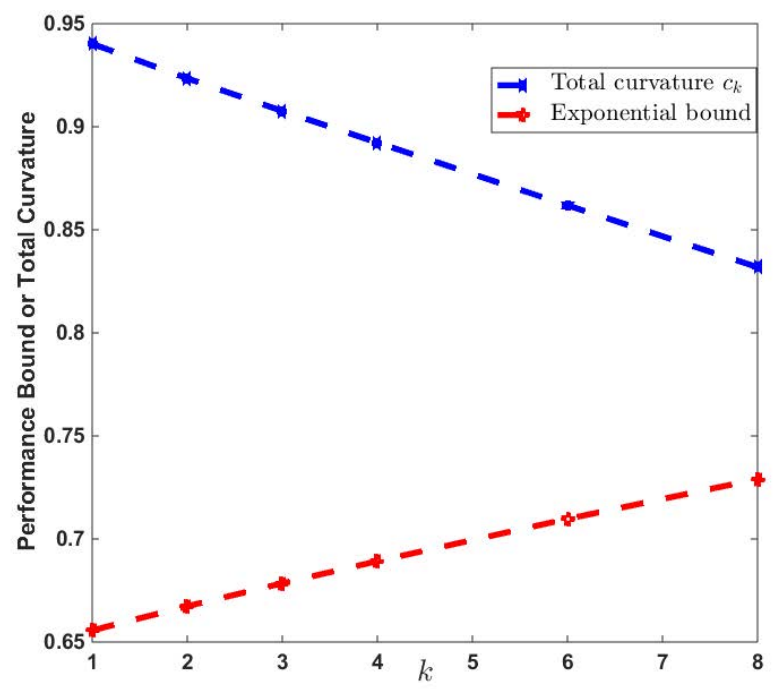

Fig. 4 Total curvature/performance bound for greedy strategy in adaptive sensing problem

\subsection{Noncooperative Games}

In the previous sections, we reviewed performance bounds for greedy-type strategies in set submodular optimization problems. It turns out that similar techniques can be used to bound the performance of Nash equilibria in noncooperative games-utility maximization problems. The connection to the game setting is easy to imagine by associating the objective function in set optimization with a social utility function in games, greedy strategies with Nash equilibria, and batching with cooperation of subgroups in games. We first introduce some background on utility maximization problems and Nash equilibria.

A great number of interesting practical problems can be posed as utility maximization problems: these include facility location Ahmed and Atamtürk 2011), traffic routing and congestion management (Arslan et al. 2007: He et al. 2007), sensor selection (Rowaihy et al. 2007; Liu et al.|2014), and network resource allocation (La and Anantharam 2002; Palomar and Chiang 2007). In a utility maximization problem, a set of users make decisions according to their own set of feasible strategies, resulting in an overall social utility value, such as profit, coverage, achieved data rate, and quality of service. The goal is to maximize the social utility function. Often, the users do not cooperate in selecting their strategies.

In general, it is impractical to find the optimal strategy maximizing the social utility function. However, it is feasible to consider scenarios where individual users or groups of users separately maximize their own private objective functions. The usual framework for studying such scenarios is game theory to- 
gether with its celebrated notion of Nash equilibria. A Nash equilibrium is a set of strategies (deterministic or randomized) for which no user can improve its own private utility by changing its strategy unilaterally. Nash (1951) proved that any finite and non-cooperative game has at least one Nash equilibrium.

The performance of Nash equilibria compared with the optimal solution in submodular utility maximization problems was investigated by Vetta (2002). Based on the existing results, Liu et al. (2018b) established bounds for Nash equilibria when there is "grouping" among users, which is useful in understanding the role of cooperation and social ties in games. Before we review these results, we introduce some notation and terminology from Vetta (2002) and Liu et al. (2018b).

Suppose we have a set $\mathcal{N}=\{1,2, \ldots, N\}$ of $N$ users. Each element in $V_{i}(i=1, \ldots, N)$ represents an act that user $i$ can take. We call a set of acts an action, and if an action $x_{i} \subseteq V_{i}$ is available to user $i$ we call it a feasible action. We denote by $\mathcal{X}_{i}$ the set of all feasible actions for user $i$, i.e., $\mathcal{X}_{i}=\left\{x_{i} \subseteq V_{i}: x_{i}\right.$ is a feasible action $\}$, with $n_{i}=\left|\mathcal{X}_{i}\right|$ the cardinality of $\mathcal{X}_{i}$. We call $\mathcal{X}_{i}$ the action space for user $i$. A pure strategy is one in which the user takes a specific action. A mixed strategy is one in which the user takes actions according to some probability distribution. The set of mixed strategies is called the strategy space. We represent the strategy space for user $i$ by $\mathcal{S}_{i}=\left\{s_{i} \in \mathbb{R}^{n_{i}}: \sum_{j=1}^{n_{i}} s_{i}^{j}=1, s_{i}^{j} \geq 0\right\}$, where $s_{i}=\left(s_{i}^{1}, \ldots, s_{i}^{n_{i}}\right)$ is called a strategy taken by user $i$ and $s_{i}^{j} \geq 0$ is the probability with which user $i$ takes action $j$. When $s_{i}^{j}=1$ for some $j$ and $s_{i}^{l}=0$ for all $l \neq j$, user $i$ is said to take a pure strategy. Otherwise, user $i$ takes a mixed strategy. Write $\mathcal{S}=\prod_{i=1}^{N} \mathcal{S}_{i}$. The indexed set $S=\left(s_{1}, \ldots, s_{N}\right)$, with $s_{i} \in \mathcal{S}_{i}$ and $i=1, \ldots, N$, is called a strategy set of size $N$ in $\mathcal{S}$.

Given a strategy set $S=\left(s_{1}, \ldots, s_{N}\right) \in \mathcal{S}$, the set $S_{-i}=$ $\left(s_{1}, \ldots, s_{i-1}, s_{i+1}, \ldots, s_{N}\right)$ is the subset of $S$ that contains strategies taken by all users except user $i$, and $\left(S_{-i}, s_{i}^{\prime}\right)=\left(s_{1}, \ldots, s_{i-1}, s_{i}^{\prime}, s_{i+1}, \ldots, s_{N}\right)$ is the strategy set that results from $S$ when user $i$ changes its strategy from $s_{i}$ to $s_{i}^{\prime}$.

The expected social utility function and expected private utility function for user $i$ from strategies in $\mathcal{S}$ to real numbers are denoted by $\bar{\gamma}$ and $\bar{\alpha}_{i}$, respectively. Define $\bar{\gamma}_{s_{i}}\left(S_{-i}\right)=\bar{\gamma}(S)-\bar{\gamma}\left(S_{-i}\right)$ for any set $S=\left(s_{1}, \ldots, s_{N}\right) \in \mathcal{S}$ and $s_{i}(i=1, \ldots, N)$.

Now we introduce the definition of a Nash equilibrium and a valid system, then review performance bounds for Nash equilibria under some conditions from Vetta (2002).

Definition 1 A strategy set $S \in \mathcal{S}$ is a Nash equilibrium if no user has an incentive to unilaterally change its strategy, i.e., for any user $i$,

$$
\bar{\alpha}_{i}(S) \geq \bar{\alpha}_{i}\left(\left(S_{-i}, s_{i}^{\prime}\right)\right), \quad \forall s_{i}^{\prime} \in \mathcal{S}_{i}
$$

Assumption 1 (Vetta 2002) The private utility of user $i(i=1, \ldots, N)$ is at least as large as the loss in the social utility resulting from user $i$ dropping 
out of the game. That is, the system $\left(\bar{\gamma},\left\{\bar{\alpha}_{i}\right\}_{i=1}^{N}\right)$ has the property that for any strategy set $S=\left(s_{1}, \ldots, s_{N}\right) \in \mathcal{S}$,

$$
\bar{\alpha}_{i}(S) \geq \bar{\gamma}_{s_{i}}\left(S_{-i}\right), \quad \forall i=1, \ldots, N .
$$

Assumption 2 (Vetta 2002) The sum of the private utilities of the system is not larger than the social utility, i.e., for any strategy set $S=\left(s_{1}, \ldots, s_{N}\right) \in \mathcal{S}$,

$$
\sum_{i=1}^{N} \bar{\alpha}_{i}(S) \leq \bar{\gamma}(S)
$$

A utility system $\left(\bar{\gamma},\left\{\bar{\alpha}_{i}\right\}_{i=1}^{N}\right)$ satisfying Assumptions 1 and 2 is called a valid system. We denote by $\Omega=\left(\omega_{1}, \ldots, \omega_{N}\right)$ the optimal strategy set in maximizing an expected utility function $\bar{\gamma}$, and assume that $\Omega$ is composed of pure strategies $\omega_{i} \in \mathcal{S}_{i}, i=1, \ldots, N$. For convenience, we also use $\omega_{i}$ to denote the optimal action that user $i$ takes. Consider a strategy set $S=\left(s_{1}, \ldots, s_{i}\right)$ where $i=1, \ldots, N$. Suppose that user $j(j=1, \ldots, i)$ uses a mixed strategy $s_{j}$ that takes actions $x_{j}^{1}, \ldots, x_{j}^{n_{j}}$ with probabilities $s_{j}^{1}, \ldots, s_{j}^{n_{j}}$. We use the notation $\Omega \cup S$ to represent the strategy in which user $j(j=1, \ldots, i)$ takes the actions $\omega_{j} \cup x_{j}^{1}, \ldots, \omega_{j} \cup x_{j}^{n_{j}}$ with probabilities $s_{j}^{1}, \ldots, s_{j}^{n_{j}}$, and user $j$ $(j=i+1, \ldots, N)$ takes the action $\omega_{j}$, so $\Omega \cup S$ is well defined.

Theorem 12 (Vetta 2002) For a valid utility system $\left(\bar{\gamma},\left\{\bar{\alpha}_{i}\right\}_{i=1}^{N}\right)$, if the expected social utility function $\bar{\gamma}$ is submodular, then for any Nash equilibrium $S \in \mathcal{S}$ we have

$$
\bar{\gamma}(S) \geq \frac{1}{2}\left(\bar{\gamma}(\Omega)+\sum_{i=1}^{N} \bar{\gamma}_{s_{i}}\left(\Omega \cup S_{-i}\right)\right) .
$$

Remark 20 If $\bar{\gamma}$ is monotone, then $\bar{\gamma}_{s_{i}}\left(\Omega \cup S_{-i}\right) \geq 0$ and the above inequality shows that any Nash equilibrium achieves at least $1 / 2$ of the optimal social utility function value.

By defining the curvature $c$ of the expected social utility function $\bar{\gamma}$,

$$
c:=\max _{i: \bar{\gamma}_{s_{i}}(\emptyset) \neq 0}\left\{1-\frac{\bar{\gamma}_{s_{i}}\left(\Omega \cup S_{-i}\right)}{\bar{\gamma}_{s_{i}}(\emptyset)}\right\},
$$

Vetta (2002) derived the following tighter performance bound in terms of the curvature for Nash equilibria.

Theorem 13 Vetta 2002) For a valid utility system $\left(\bar{\gamma},\left\{\bar{\alpha}_{i}\right\}_{i=1}^{N}\right)$, if the expected social utility function $\bar{\gamma}$ is monotone and submodular, then for any Nash equilibrium $S \in \mathcal{S}$ we have

$$
\frac{\bar{\gamma}(S)}{\bar{\gamma}(\Omega)} \geq \frac{1}{1+c} .
$$

Remark 21 When the expected social utility function $\bar{\gamma}$ is monotone and submodular, we have $c \in[0,1]$, which implies that $\bar{\gamma}(S) \geq \bar{\gamma}(\Omega) / 2$. 
Next we review performance bounds for group Nash equilibria defined by Liu et al. (2018b). They considered the case where the set of all users in the utility maximization system are divided into disjoint groups, and the users in the same group choose their strategies by maximizing their group utility function jointly.

Assume that the set of users $\mathcal{N}=\{1, \ldots, N\}$ is divided into $l$ disjoint groups, in which group $i(i=1, \ldots, l)$ has users $\left\{m_{i}+1, \ldots, m_{i}+k_{i}\right\}$, where $m_{i}=\sum_{j=1}^{i-1} k_{j}, k_{j}$ is the number of users in group $j$, and $\sum_{j=1}^{l} k_{j}=N$. Let $s^{i}=\left(s_{m_{i}+1}, \ldots, s_{m_{i}+k_{i}}\right)$ denote the group strategy for group $i$, where $s_{i} \in \mathcal{S}_{i}$ is the strategy for user $i$. This includes the strategies taken by all the users in group $i(i=1, \ldots, l)$. Let $S^{-i}$ denote the set of group strategies taken by all groups except for group $i$ and $\left(S^{-i}, t^{i}\right)$ denote the group strategy set obtained when group $i$ changes its group strategy from $s^{i}$ to $t^{i}$. Let $\bar{\eta}_{i}$ denote the expected group utility function for group $i$. Define $\bar{\gamma}_{s^{i}}\left(S^{-i}\right)=\bar{\gamma}(S)-\bar{\gamma}\left(S^{-i}\right)$ for any $S=\left(s^{1}, \ldots, s^{l}\right) \in \mathcal{S}$ and $s^{i}(i=1, \ldots, l)$.

Definition 2 A strategy set $S=\left(s^{1}, \ldots, s^{l}\right) \in \mathcal{S}$ is a group Nash equilibrium of a utility system if no group can improve its group utility by unilaterally changing its group strategy, i.e., for any $i=1, \ldots, l$,

$$
\bar{\eta}_{i}(S) \geq \bar{\eta}_{i}\left(\left(S^{-i}, t^{i}\right)\right), \quad \forall t^{i}=\left(t_{m_{i}+1}, \ldots, t_{m_{i}+k_{i}}\right),
$$

where $t_{j} \in \mathcal{S}_{j}$ for $j=m_{i}+1, \ldots, m_{i}+k_{i}$.

The utility system $\left(\bar{\gamma},\left\{\bar{\eta}_{i}\right\}_{i=1}^{l}\right)$ is valid if it satisfies the following two assumptions (Liu et al. 2018b).

Assumption 3 The group utility of group $i$ is at least as large as the loss in the social utility resulting from all the users in group $i$ dropping out of the game. That is, the system $\left(\bar{\gamma},\left\{\bar{\eta}_{i}\right\}_{i=1}^{l}\right)$ has the property that for any strategy set $S=\left(s^{1}, \ldots, s^{l}\right) \in \mathcal{S}$,

$$
\bar{\eta}_{i}(S) \geq \bar{\gamma}_{s^{i}}\left(S^{-i}\right), \quad \forall i=1, \ldots, l .
$$

Assumption 4 The sum of the group utilities of the system is not larger than the social utility, i.e., for any strategy set $S=\left(s^{1}, \ldots, s^{l}\right) \in \mathcal{S}$,

$$
\sum_{i=1}^{l} \bar{\eta}_{i}(S) \leq \bar{\gamma}(S)
$$

Theorem 14 (Liu et al. 2018b) For a valid utility system $\left(\bar{\gamma},\left\{\bar{\eta}_{i}\right\}_{i=1}^{l}\right)$, if the expected social utility function $\bar{\gamma}$ is submodular, then any group Nash equilibrium $S=\left(s^{1}, \ldots, s^{l}\right) \in \mathcal{S}$ satisfies

$$
\bar{\gamma}(S) \geq \frac{1}{2}\left(\bar{\gamma}(\Omega)+\sum_{i=1}^{l} \bar{\gamma}_{s^{i}}\left(\Omega \cup S^{-i}\right)\right) .
$$


To better characterize the relation of the social utility value of any group Nash equilibrium and that of the optimal solution $\Omega$, Liu et al. (2018b) defined the group curvature $c_{k_{i}}$ of the social utility function for group $i$ as

$$
c_{k_{i}}:=\max _{S \in \mathcal{S}, \bar{\gamma}_{s^{i}}(\emptyset) \neq 0}\left\{1-\frac{\bar{\gamma}_{s^{i}}\left(\Omega \cup S^{-i}\right)}{\bar{\gamma}_{s^{i}}(\emptyset)}\right\} .
$$

Theorem 15 Liu et al. 2018b) For a valid utility system $\left(\bar{\gamma},\left\{\bar{\eta}_{i}\right\}_{i=1}^{l}\right)$, if the expected social utility function $\bar{\gamma}$ is monotone and submodular, then any group Nash equilibrium $S=\left(s^{1}, \ldots, s^{l}\right) \in \mathcal{S}$ satisfies

$$
\bar{\gamma}(S) \geq \frac{1}{1+\max _{1 \leq i \leq l} c_{k_{i}}} \bar{\gamma}(\Omega) .
$$

In particular, if $\mathcal{X}_{1}=\mathcal{X}_{2}=\cdots=\mathcal{X}_{N}$, we have

$$
\bar{\gamma}(S) \geq \frac{1}{1+c_{k^{*}}} \bar{\gamma}(\Omega)
$$

where $k^{*}=\min _{1 \leq i \leq l} k_{i}$.

Remark 22 When the expected group utility function $\bar{\gamma}$ is monotone and submodular, it is easy to check that $c_{k_{i}} \in[0,1]$, which implies that $1 /(1+$ $\left.\max _{1 \leq i \leq l} c_{k_{i}}\right) \geq 1 / 2$.

Remark 23 When the expected group utility function $\bar{\gamma}$ is monotone and submodular, we have $\bar{\gamma}(S) \geq \bar{\gamma}(\Omega) /\left(1+\max _{1 \leq i \leq l} c_{k_{i}}\right) \geq \bar{\gamma}(\Omega) /(1+c)$. This shows that the bound for the case with grouping is tighter than that for the case without grouping. Of course, this is unsurprising, because grouping entails cooperation. Moreover, under the condition that each user has the same strategy space, the larger the value of $k_{i}$, the higher the degree of cooperation, and the tighter the lower bound.

\section{Strings of Actions}

In Section 2, we considered the optimization problem where the argument of the objective function is a set of actions. Suppose the objective function depends not only on the set of actions but also on the order of actions. We call the argument of the objective function a string of actions. In this section, we introduce notation and terminology for strings and string functions, string optimization problem, performance bounds for the greedy strategy, and applications. 


\subsection{Notation and Terminology}

Let $X$ be a set of all possible actions. We use $A=\left(a_{1}, a_{2}, \ldots, a_{k}\right)\left(a_{i} \in X\right)$ to denote a string of actions taken over $k$ consecutive stages. We define its length as $k$, denoted by $|A|=k$. Note that $k=0$ corresponds to the empty string, denoted by $A=\emptyset$.

Let $X^{*}$ denote the set of all possible strings of actions. If two strings in $X^{*}$ are expressed by $M=\left(a_{1}^{m}, a_{2}^{m}, \ldots, a_{k_{1}}^{m}\right)$ and $N=\left(a_{1}^{n}, a_{2}^{n}, \ldots, a_{k_{2}}^{n}\right)$, we write $M=N$ iff $k_{1}=k_{2}$ and $a_{i}^{m}=a_{i}^{n}$ for each $i=1,2, \ldots, k_{1}$. Moreover, we define string concatenation as $M \oplus N=\left(a_{1}^{m}, a_{2}^{m}, \ldots, a_{k_{1}}^{m}, a_{1}^{n}, a_{2}^{n}, \ldots, a_{k_{2}}^{n}\right)$.

We write $M \preceq N$ if we have $N=M \oplus L$ for some $L \in X^{*}$. In this case, we also say that $M$ is a prefix of $N$. We write $M \prec N$ if there exists a set of strings $L_{i} \in X^{*}$ such that $N=L_{1} \oplus\left(a_{1}^{m}, \ldots, a_{i_{1}}^{m}\right) \oplus L_{2} \oplus\left(a_{i_{1}+1}^{m}, \ldots, a_{i_{2}}^{m}\right) \oplus \cdots \oplus$ $\left(a_{i_{k-1}+1}^{m}, \ldots, a_{k_{1}}^{m}\right) \oplus L_{k}$. Note that $\prec$ is weaker than $\preceq$, which means $M \preceq N$ implies $M \prec N$, but the converse is not necessarily true.

Similar to the definition of a polymatroid set function in Section 2.1, we define a function from strings to real numbers, $f: X^{*} \rightarrow \mathbb{R}$, a polymatroid string function if

i. $f(\emptyset)=0$.

ii. $f$ has the prefix-monotone property: $\forall M, N \in X^{*}, f(M \oplus N) \geq f(M)$.

iii. $f$ has the diminishing-return property: $\forall M \preceq N \in X^{*}, \forall a \in X, f(M \oplus$ $(a))-f(M) \geq f(N \oplus(a))-f(N)$.

A function $f: X^{*} \rightarrow \mathbb{R}$ is postfix monotone if

$$
\forall M, N \in X^{*}, f(M \oplus N) \geq f(N) .
$$

Notice the difference between the prefix-monotone property and postfixmonotone property.

Let $\mathcal{I}$ denote a collection of strings from $X^{*}$. The pair $(X, \mathcal{I})$ is called a string matroid (Zhang et al. 2016) if $\mathcal{I}$ satisfies the following properties:

i. $\mathcal{I}$ is non-empty;

ii. Hereditary: $\forall M \in \mathcal{I}, N \prec M$ implies that $n \in \mathcal{I}$;

iii. Augmentation: $\forall M, N \in \mathcal{I}$ and $|M|<|N|$, there exists an element $x \in X$ in the string $N$ such that $M \oplus(x) \in \mathcal{I}$.

The length of the longest string in $\mathcal{I}$ is called the rank of $(X, \mathcal{I})$. When $\mathcal{I}=$ $\left\{A \in X^{*}:|A| \leq K\right\}$, the pair $(X, \mathcal{I})$ is called a uniform string matroid of rank $K$.

\subsection{String Optimization Problem}

In this section, we first formulate the string optimization problem and define the greedy strategy. Then we review performance bounds for the greedy strategy under uniform string matroid constraints and general string matroid constraints. 
In a variety of problems in engineering and applied science such as sequential decision making (Littman 1996, Roijers et al. 2013), adaptive sensing (Liu et al. 2014, Krause et al. 2008), and adaptive control (Jarvis 1975, Schlegel et al. 2005), we are faced with optimally choosing a string (ordered set) of actions over a finite horizon to maximize an objective function under some constraints. We call this class of optimization problems string optimization. For set optimization problems, the objective function is not influenced by the order of actions. However, for string optimization problems, the objective function depends on the order of actions. Let $f: X^{*} \rightarrow \mathbb{R}$ be an objective function. The goal is to find a string $M$, with the constraint $M \in \mathcal{I}$, to maximize the objective function:

$$
\text { maximize } f(M), \quad \text { subject to } M \in \mathcal{I} \text {, }
$$

where $X^{*}$ denotes the set of all possible strings of actions and $\mathcal{I}$ is a collection of strings from $X^{*}$.

The solution to the string optimization problems can be characterized using dynamic programming via Bellman's principle (Bertsekas 2005, Powell 2007). However, dynamic programming suffers from the curse of dimensionality and is therefore impractical for many problems of interest. Hence, we often turn to approximation techniques. One approximation technique is the greedy strategy, which is to find an action at each stage to maximize the step-wise gain in the objective function. The performance for the greedy strategy in string optimization problems has been investigated by Streeter and Golovin (2008), Zhang et al. (2016), and Liu et al. (2015). And these specific results will be reviewed in this section.

Assume that the rank of $(X, \mathcal{I})$ is $K$. We now define optimal and greedy strategies for problem (28) and some related notation.

Optimal String: Any string $O$ is called an optimal solution of Problem (28) if

$$
O \in \underset{M \in \mathcal{I}}{\operatorname{argmax}} f(M) .
$$

If $f$ is prefix monotone, then there exists at least one optimal string of length $K$, denoted by $O_{K}=\left(o_{1}, \ldots, o_{K}\right)$. 
Greedy Algorithm:

Input: A string matroid $(X, \mathcal{I})$ of rank $K$, a set function $f: X^{*} \rightarrow \mathbb{R}$, collection $\mathcal{I}$, size $K$

Output: A string $G_{K} \in \mathcal{I}$

$G_{0} \leftarrow \emptyset$

For $i=1, \ldots, K$,

1. $g_{i} \leftarrow \underset{a \in X, G_{i-1} \oplus(a) \in \mathcal{I}}{\operatorname{argmax}} f\left(G_{i-1} \oplus(a)\right)$

2. $G_{i} \leftarrow G_{i-1} \oplus\left(g_{i}\right)$

Any output of the above algorithm is called a greedy solution. There may exist more than one greedy solution.

\subsection{Performance Bounds for Greedy Strategy}

Streeter and Golovin (2008) first derived performance bounds for the greedy strategy under uniform string matroid constraints, stated as follows.

Theorem 16 (Streeter and Golovin 2008) Let $(X, \mathcal{I})$ be a uniform string matroid. If $f: X^{*} \rightarrow \mathbb{R}$ is a polymatroid string function and postfix monotone, then any greedy string $G_{K}$ satisfies

$$
\frac{f\left(G_{K}\right)}{f\left(O_{K}\right)} \geq 1-\left(1-\frac{1}{K}\right)^{K}>1-e^{-1} .
$$

Remark 24 The same bound holds if $f$ satisfies $f\left(G_{i} \oplus O_{K}\right) \geq f\left(O_{K}\right)$ for $i=1, \ldots, K-1$, which is weaker than being postfix monotone.

Zhang et al. (2016) investigated performance bounds for the greedy strategy under both uniform string matroid and general string matroid constraints by defining the following curvatures.

The total backward curvature of $f$ is defined as (Zhang et al. 2016)

$$
\sigma:=\max _{\substack{a \in X, M \in X^{*} \\ f((a)) \neq f(\emptyset)}}\left\{1-\frac{f((a) \oplus M)-f(M)}{f((a))-f(\emptyset)}\right\} .
$$

When $f$ is postfix monotone and diminishing return, we have $0 \leq \sigma \leq 1$. The total backward curvature is an upper bound on the second-order difference, over all possible actions $a$ and strings $M$. Next, Zhang et al. (2016) defined the total backward curvature of $f$ with respect to the optimal string $O_{K}$ by

$$
\sigma\left(O_{K}\right):=\max _{\substack{N \in X^{*}, 0<|N| \leq K \\ f(N) \neq f(\emptyset)}}\left\{1-\frac{f\left(N \oplus O_{K}\right)-f\left(O_{K}\right)}{f(N)-f(\emptyset)}\right\} .
$$

When $f$ is postfix monotone and string submodular, it is easy to prove that $0 \leq \sigma(O) \leq \sigma \leq 1$. 
Theorem 17 (Zhang et al. 2016) Let $(X, \mathcal{I})$ be a uniform string matroid of rank $K$. If $f: X^{*} \rightarrow \mathbb{R}$ is a polymatroid string function, then any greedy string $G_{K}$ satisfies

$$
\frac{f\left(G_{K}\right)}{f\left(O_{K}\right)} \geq \frac{1}{\sigma\left(O_{K}\right)}\left[1-\left(1-\frac{\sigma\left(O_{K}\right)}{K}\right)^{K}\right]>\frac{1}{\sigma\left(O_{K}\right)}\left(1-e^{-\sigma\left(O_{K}\right)}\right) .
$$

Moreover, if $f$ is postfix monotone, then any greedy string $G_{K}$ satisfies

$$
\frac{f\left(G_{K}\right)}{f\left(O_{K}\right)} \geq \frac{1}{\sigma}\left[1-\left(1-\frac{\sigma}{K}\right)^{K}\right]>\frac{1}{\sigma}\left(1-e^{-\sigma}\right) .
$$

Remark 25 When $f$ is polymatroid and postfix monotone, we have $0 \leq \sigma \leq 1$ by 30 . So we have $\left(1-(1-\sigma / K)^{K}\right) / \sigma \geq 1-(1-1 / K)^{K}$ and $\left(1-e^{-\sigma}\right) / \sigma>1-$ $e^{-1}$, which implies that Theorem 17 provides better bounds than Theorem 16 .

Theorem 18 (Zhang et al. $\mid$ 2016) Let $(X, \mathcal{I})$ be a string matroid. If $f: X^{*} \rightarrow$ $\mathbb{R}$ is a polymatroid string function, then any greedy string $G_{K}$ satisfies

$$
\frac{f\left(G_{K}\right)}{f\left(O_{K}\right)} \geq \frac{1}{1+\sigma\left(O_{K}\right)} .
$$

Moreover, if $f$ is postfix monotone, then any greedy string $G_{K}$ satisfies

$$
\frac{f\left(G_{K}\right)}{f\left(O_{K}\right)} \geq \frac{1}{1+\sigma} .
$$

From Theorems 16 and 17, we can see that all the sufficient conditions obtained so far involve strings of length greater than $K$, even though (28) involves only strings up to length $K$. Liu et al. (2015) derived sufficient conditions, which only involve strings of length at most $K$, to have the same bounds hold for uniform string matroid constraints, by defining the following conditions.

A function $f: X^{*} \rightarrow \mathbb{R}$ is $K$-polymatroid if

i. $f(\emptyset)=0$.

ii. $f$ is $K$-monotone: $\forall M, N \in X^{*}$, and $|M|+|N| \leq K, f(M \oplus N) \geq f(M)$.

iii. $f$ is $K$-diminishing: $\forall M \preceq N \in X^{*}$ and $|N| \leq K-1, \forall a \in X, f(M \oplus$ $(a))-f(M) \geq f(N \oplus(a))-f(N)$.

Let $G_{K}=\left(g_{1}, \ldots, g_{K}\right)$ and $\bar{O}_{K-i}=\left(o_{i+1}, \ldots, o_{K}\right)$ for $i=1, \ldots, K$. Then, $f$ is $K$-GO-concave (Liu et al. 2015) if for $1 \leq i \leq K-1$,

$$
f\left(G_{i} \oplus \bar{O}_{K-i}\right) \geq \frac{i}{K} f\left(G_{i}\right)+\left(1-\frac{i}{K}\right) f\left(O_{K}\right) .
$$

Theorem 19 Liu et al. 2015) Let $(X, \mathcal{I})$ be a uniform string matroid. If $f$ is $K$-polymatroid, then any greedy string satisfies

$$
\frac{f\left(G_{K}\right)}{f\left(O_{K}\right)} \geq\left[1-\left(1-\frac{1}{K}\right)^{K}\right]>\left(1-e^{-1}\right) .
$$


By defining the curvature $\eta$,

$$
\eta=\max _{1 \leq i \leq K-1}\left\{\frac{K f\left(G_{i}\right)-\left(K f\left(G_{i} \oplus \bar{O}_{K-i}\right)-(K-i) f\left(O_{K}\right)\right)}{(K-i) f\left(G_{i}\right)}\right\},
$$

Liu et al. (2015) derived more general performance bounds in terms of the curvature.

Theorem 20 (Liu et al. 2015) Let $(X, \mathcal{I})$ be a uniform string matroid. If $f$ is $K$-polymatroid and $K$-GO-concave, then any greedy string satisfies

$$
\frac{f\left(G_{K}\right)}{f\left(O_{K}\right)} \geq \frac{1}{\eta}\left[1-\left(1-\frac{\eta}{K}\right)^{K}\right]>\frac{1}{\eta}\left(1-e^{-\eta}\right) .
$$

Remark 26 If $f$ is $K$-GO-concave, then we have $0 \leq \eta \leq 1$.

Examples: We again consider the task assignment problem and adaptive sensing problem from Section 2.4 to give some sufficient conditions on the parameters of the problems to achieve the performance bound $\left(1-(1-1 / K)^{K}\right)$.

Task Assignment Problem: We use $p_{i}^{j}(a)$ to denote the probability of accomplishing subtask $i$ at stage $j$ when it is assigned to agent $a \in X$. Let $a_{j}$ be the index of the agent selected at stage $j$, the objective function $f$ becomes

$$
f\left(\left(a_{1}, \ldots, a_{k}\right)\right)=\frac{1}{n} \sum_{i=1}^{n}\left(1-\prod_{j=1}^{k}\left(1-p_{i}^{j}\left(a_{j}\right)\right)\right) .
$$

For simplicity, we consider the case of $n=1$ (our results can easily be generalized to the case where $n>1$ ). For $n=1$, the objective function $f$ reduces to

$$
f\left(\left(a_{1}, \ldots, a_{k}\right)\right)=1-\prod_{j=1}^{k}\left(1-p_{1}^{j}\left(a_{j}\right)\right),
$$

and from here on we simply use $p^{j}\left(a_{j}\right)$ in place of $p_{1}^{j}\left(a_{j}\right)$.

Note that the value of $f$ depends on the order of the agents selected over time when the probabilities vary from stage to stage. In other words, suppose that we have two agents, Alice and Bob. Then, in general, $p^{1}$ (Alice) $\neq$ $p^{2}$ (Alice), $p^{1}(\mathrm{Bob}) \neq p^{2}(\mathrm{Bob}), p^{1}$ (Alice) $\neq p^{1}(\mathrm{Bob})$, and $p^{2}$ (Alice) $\neq p^{2}(\mathrm{Bob})$. Therefore, $f(($ Alice, Bob $)) \neq f(($ Bob, Alice $))$.

It is easy to check that $f$ is $K$-monotone and $f(\emptyset)=0$.

Assume that $p^{j}(a) \in[L(a), U(a)]$, where $L(a)=\min _{j} p^{j}(a)$ and $U(a)=$ $\max _{j} p^{j}(a)$. By Zhang et al. (2016), a sufficient condition for $f$ to be diminishing return is

$$
p^{1}\left(g_{1}\right) \geq 1-c^{K},
$$

where

$$
c=\min _{a \in \mathbb{A}} \frac{1-U(a)}{1-L(a)} .
$$


Let $\hat{U}=\max _{a} U(a)$ and $\hat{L}=\min _{a} L(a)$. By Liu et al. (2015), a sufficient condition for $f$ to be $K$-diminishing is

$$
\hat{L} \geq(1-\hat{L}) \hat{U},
$$

and a sufficient condition for $K$-GO-concavity is

$$
\hat{L} \geq \frac{1}{2}
$$

When all $p^{j}\left(a_{j}\right) \geq 1 / 2$, then (39) and (38) automatically hold, but (37) is not necessarily satisfied. In that sense, the $K$-monotone, $K$-diminishing, and $K$-Go concavity conditions of Theorem 19 are weaker sufficient conditions for achieving the bound $\left(1-\left(1-\frac{1}{K}\right)^{K}\right)$ than the prefix monotone, diminishingreturn, and postfix monotone conditions of Theorem 16

Adaptive Sensing: Consider the situation where the additive noise set is independent but not identically distributed. Assume that $w_{i}$ is a Gaussian vector with mean zero and covariance $\sigma_{i} I$, where $I$ denotes the identity matrix. Recall the problem formulation in Section 2.4. The objective function $f$ for this problem is as follows:

$$
f\left(\left(B_{1}, \ldots, B_{k}\right)\right)=\frac{1}{2}\left(\log \operatorname{det}\left(P_{0}\right)-\log \operatorname{det}\left(P_{k}\right)\right) .
$$

where $P_{0}=I$ and for $1 \leq j \leq k-1$,

$$
P_{j}=\left(P_{j-1}^{-1}+\frac{1}{\sigma_{j}^{2}} B_{j}^{T} B_{j}\right)^{-1} .
$$

From the expression above, it is easy to check that the order of $B_{1}, \ldots, B_{k}$ influences the objective function value under the assumption that $\sigma_{1}, \ldots, \sigma_{k}$ take different values. For example,

$$
f((A, B))=\frac{1}{2} \log \operatorname{det}\left(I+\frac{1}{\sigma_{1}^{2}} A^{T} A+\frac{1}{\sigma_{2}^{2}} B^{T} B\right)
$$

and

$$
f((B, A))=\frac{1}{2} \log \operatorname{det}\left(I+\frac{1}{\sigma_{1}^{2}} B^{T} B+\frac{1}{\sigma_{2}^{2}} A^{T} A\right) .
$$

If $\sigma_{1} \neq \sigma_{2}$, then $f((A, B)) \neq f((B, A))$.

By Liu et al. (2015), some sufficient conditions for $f$ to be $K$-polymatroid and $K$-GO-concave are

$$
\sigma_{i+1}^{2} \geq \sigma_{i}^{2}
$$

for $i=1, \ldots, K-1$.

By Zhang et al. (2016), to achieve the bound $\left(1-(1-1 / K)^{K}\right)$, it requires both 400 and

$$
\frac{b^{-2}}{a^{-2}-b^{-2}} \geq(K-1)^{2}\left(a^{-2}+b^{-2}\right)+1,
$$

where $[a, b]$ is the interval that contains all the $\sigma_{i}$ 's.

Comparing the sufficient conditions for achieving the same bound (1$\left.(1-1 / K)^{K}\right)$ from Liu et al. (2015) and Zhang et al. (2016), we see that the conditions from Liu et al. (2015) are weaker. 


\section{Final Remarks}

In this survey, we considered two classes of submodular maximization problems: set submodular maximization and string submodular maximization. For set submodular optimization, we reviewed performance bounds for the greedy strategy under matroid constraints, improved performance bounds, and performance bounds for the batched greedy strategy. There are many important results about performance of the greedy strategy under some other constraints and conditions. Wolsey (1982), Sviridenko (2004), and Kulik et al. (2009) derived performance bounds for the greedy strategy in submodular maximization problems subject to a knapsack constraint and multiple linear constraints. Bian et al. (2017) established performance bounds for the greedy strategy in monotone but nonsubmodular maximization problems under uniform matroid constraints. People also investigated performance bounds for some variations of greedy strategies. Calinescu et al. (2011) and Feldman et al. (2011) derived performance bounds for a randomized continuous greedy algorithm and a unified continuous greedy algorithm in monotone submodular maximization problems, respectively. Buchbinder et al. (2012) established performance bounds for an adaptive greedy algorithm in unconstrained submodular maximization problems. They also derived performance bounds for randomized greedy algorithms in nonmonotone submodular maximization problems (Buchbinder et al. 2014). Mirzasoleiman et al. (2016) considered submodular maximization problems in a distributed fashion, and they derived performance bounds for a two-stage greedy algorithm under matroid or knapsack constraints. Qu et al. (2015) proposed a distributed greedy strategy and showed that it has the same guarantee as the centralized greedy strategy.

For string submodular optimization problems, we reviewed performance bounds for the greedy strategy under matroid constraints. There are some related results on performance bounds for greedy strategies in string submodular maximization problems that were not reviewed in this paper. For example, Golovin and Krause (2001) considered a particular class of partially observable adaptive stochastic optimization problems, and established performance bounds for the greedy strategy by introducing the notion of adaptive submodularity. Tschiatschek et al. (2017) derived performance bounds for a modified greedy strategy in submodular string optimization problems under uniform string matroid constraints.

The scope of this study is limited to the performance of the greedy strategies in deterministic optimization problems where the objective function only involves actions. Potentially fruitful areas for further research include performance bounds for the greedy strategy in stochastic optimization problems, where the objective function involves states and control actions, and real-world applications of the performance bounds in the deterministic and stochastic settings. 


\section{References}

Ahmed, S., and A. Atamtürk, 2011: Maximizing a class of submodular utility functions. Math Program, 128, 149-169.

Arslan, G., J. R. Marden, and J. S. Shamma, 2007: Autonomous vehicle-target assignment: a game-theoretical formulation. J Dyn Syst Meas Control, 129, $584-596$

Badanidiyuru, A., B. Mirzasoleiman, A. Karbasi, and A. Krause, 2014: Streaming submodular maximization: massive data summarization on the fly. In: Proceedings of the 20th ACM SIGKDD International Conference on Knowledge Discovery and Data Mining, 671-680.

Bator, F. M., 1957: The simple analytics of welfare maximization. Am Econ Rev, 47, 22-59.

Bertsekas, D. P., 2005: Dynamic programming and optimal control. 3rd ed., Athena Scientific.

Bian, A. A., J. M. Buhmann, A. Krause, and S. Tschiatschek, 2017: Guarantee for greedy maximization of non-submodular functions with applications. In: Proceedings of the 34th International Conference on Machine Learning, 498507.

Boros, E., K. Elbassioni, and L. Khachiyan, 2003: An inequality for polymatroid functions and its applications. Discrete Appl Math, 131, 255-281.

Buchbinder, N., M. Feldman, J. Naor, and R. Schwartz, 2012: A tight linear time (1/2)-approximation for unconstrained submodular maximization. SIAM J Comput, 44, 255-281.

Buchbinder, N., M. Feldman, J. Naor, and R. Schwartz, 2014: Submodular maximization with cardinality constraints. In: Proceedings of the 25th annual ACM-SIAM symposium on Discrete Algorithms, 1433-1452.

Calinescu, G., C. Chekuri, M. Pál, and J. Vondrák, 2011: Maximizing a submodular set function subject to a matroid constraint. SIAM J Comput, 40, 1740-1766.

Church, R., and C. R. Velle, 1974: The maximal covering location problem. Pap Reg Sci, 32, 101-118.

Clark, A., and R. Poovendran, 2011: A submodular optimization framework for leader selection in linear multi-agent systems. In: Proceedings of the 50th IEEE Conference on Control and Decision and European Control Conference, 3614-3621.

Cohen, R., L. Katzir, and D. Raz, 2006: An efficient approximation for the generalized assignment problem. Inf Process Lett, 100, 162-166.

Conforti, M., and G. Cornuéjols, 1984: Submodular set functions, matroids and the greedy algorithm: tight worst-case bounds and some generalizations of the Rado-Edmonds theorem. Discrete Appl Math, 7, 251-274.

Cornuéjols, G., M. L. Fisher, and G. L. Nemhauser, 1977: Location of bank accounts to optimize float: an analytic study of exact and approximate algorithms. Manag Sci, 23, 789-810.

Cunningham, W. H., 1986: Improved bounds for matroid partition and intersection algorithms. SIAM J Comput, 15, 948-957. 
Edmonds, J., 1966: Matroids and the greedy algorithm. In: Proceedings of the International Symposium on Mathematical Programming, 93-117.

Edmonds, J., 1970: Submodular functions, matroids, and certain polyhedra. Combin Structures and Their Appl, 69-87.

Edmonds, J., and D. R. Fulkerson, 1965: Transversals and matroid partition. $J$ Res Nat Bur Stand, 69B, 147-153.

Feldman, M., J. Naor, and R. Schwartz, 2011: A unified continuous greedy algorithm for submodular maximization. In: Proceedings of the 52nd IEEE Annual Symposium on Foundations of Computer Science, 570-579.

Fisher, M. L., G. L. Nemhauser, and L. A. Wolsey, 1978: An analysis of approximations for maximizing submodular set functions-II. Math Prog Study, 8, 73-87.

Fleischer, L., M. X. Goemans, V. S. Mirrokni, and M. Sviridenko, 2006: Tight approximation algorithms for maximum general assignment problems. In: Proceedings of the 17th Annual ACM-SIAM Symposium on Discrete Algorithm, 611-620.

Goemans, M. X., and D. P. Williamson, 1995: Improved approximation algorithms for maximum cut and satisfiability problems using semidefinite programming. J ACM, 42, 1115-1145.

Golovin, D., and A. Krause, 2001: Adaptive submodularity: theory and applications in active learning and stochastic optimization. J Artif Intell Res, 42, 427-486.

Hausmann, D., B. Korte, and T. A. Jenkyns, 1980: Worst case analysis of greedy type algorithms for independence systems. Math Program Stud, 12, $120-131$.

He, J., M. Bresler, M. Chiang, and J. Rexford, 2007: Towards robust multilayer traffic engineering: optimization of congestion control and routing. IEEE J Sel Area Comm, 25, 868-880.

Jarvis, R. A., 1975: Optimization strategies in adaptive control: a selective survey. IEEE Trans Syst Man Cybern, SMC-5, 83-94.

Kapralov, M., I. Post, and J. Vondrák, 2013: Online submodular welfare maximization: greedy is optimal. In: Proceedings of the 24th Annual ACM-SIAM Symposium on Discrete Algorithms, 1216-1225.

Khuller, S., A. Moss, and J. Naor, 1999: The budgeted maximum coverage problem. Inf Process Lett, 70, 39-45.

Knuth, D. E., 1973: Matroid partitioning. Technical Report, STAN-CS-73-342, Stanford University.

Korula, N., V. S. Mirrokni, and M. Zadimoghaddam, 2015: Online submodular welfare maximization: greedy beats $1 / 2$ in random order. In: Proceedings of the 47th Annual Symposium on Theory of Computing, 889-898.

Krause, A., and D. Golovin, 2012: Submodular function maximization. Tractability: Practical Approaches to Hard Problems, 3, 19.

Krause, A., A. Singh, and C. Guestrin, 2008: Near-optimal sensor placements in Gaussian processes: theory, efficient algorithms and empirical studies. $J$ Mach Learn Res, 9, 235-284. 
Kulik, A., H. Shachnai, and T. Tamir, 2009: Maximizing submodular set functions subject to multiple linear constraints. In: Proceedings of the 20th Annual ACM-SIAM Symposium on Discrete Algorithms, 545-554.

La, R. J., and V. Anantharam, 2002: Utility-based rate control in the internet for elastic traffic. IEEE/ACM Trans Netw, 10, 272-286.

Li, Y., K. L. W., E. K. P. Chong, and K. N. Groom, 2009: Approximate stochastic dynamic programming for sensor scheduling to track multiple targets. Digit Signal Process, 19, 533-545.

Lin, H., and J. Bilmes, 2011: A class of submodular functions for document summarization. In: Proceedings of the 49th Annual Meeting of the Association for Computational Linguistics: Human Language Technologies, 510520 .

Littman, M. L., 1996: Algorithms for sequential decision making. Technical Report, CS-96-09.

Liu, E., E. K. P. Chong, and L. L. Scharf, 2014: Greedy adaptive linear compression in signal-plus-noise models. IEEE Trans Inf Theory, 60, 2269-2280.

Liu, Y., E. K. P. Chong, and A. Pezeshki, 2015: Bounding the greedy strategy in finite-horizon string optimization. In: Proceedings of the 54th IEEE Conference on Decision and Control, 3900-3905.

Liu, Y., E. K. P. Chong, and A. Pezeshki, 2018a: Extending polymatroid set functions with curvature and bounding the greedy strategy. In: 2018 IEEE Statistical Signal Processing Workshop, 318-322.

Liu, Y., E. K. P. Chong, and A. Pezeshki, 2018b: Performance bounds for Nash equilibria in submodular utility systems with user groups. J Control Decision, 5, 1-18.

Liu, Y., E. K. P. Chong, and A. Pezeshki, 2019: Improved bounds for the greedy strategy in optimization problems with curvature. J Comb Optim, 37, 1126-1149.

Liu, Y., Z. Zhang, E. K. P. Chong, and A. Pezeshki, 2018c: Performance bounds for the k-batch greedy strategy in optimization problems with curvature. In: Proceedings of 2016 American Control Conference, 7177-7182.

Liu, Y., Z. Zhang, E. K. P. Chong, and A. Pezeshki, 2018d: Performance bounds with curvature for batched greedy optimization. J Optim Theory Appl, 177, 535-562.

Lovász, L., 1983: Submodular functions and convexity. Math Program, 235257.

Mirzasoleiman, B., A. Karbasi, and A. Krause, 2017: Deletion-robust submodular maximization: data summarization with "the right to be forgotten". In: Proceedings of the 34th International Conference on Machine Learning, 70, 2449-2458.

Mirzasoleiman, B., A. Karbasi, A. Sarkar, and A. Krause, 2016: Distributed submodular maximization. J Mach Learn Res, 17 (238), 1-44.

Nash, J., 1951: Non-cooperative games. Ann Math, 54, 286-295.

Nauss, R. M., 2003: Solving the generalized assignment problem: an optimizing and heuristic approach. INFORMS J Comput, 15, 249-266. 
Nemhauser, G. L., L. A. Wolsey, and M. L. Fisher, 1978: An analysis of approximations for maximizing submodular set functions-I. Math Program, 14, 265-294.

Paar, C., and J. Pelzl, 2010: Understanding cryptography: a textbook for students and practitioners. Springer.

Palomar, D. P., and M. Chiang, 2007: Alternative distributed algorithms for network utility maximization: framework and applications. IEEE Trans Autom Control, 52, 2254-2269.

Powell, W. B., 2007: Approximate dynamic programming: solving the curses of dimensionality. New York: Wiley.

Qu, G., D. Brown, and N. Li, 2015: Distributed greedy algorithm for satellite assignment problem with submodular utility function. IFAC-PapersOnLine, 48, 258-263.

Roijers, D. M., P. Vamplew, S. Whiteson, and R. Dazeley, 2013: A survey of multi-objective sequential decision-making. J Artif Intell Res, 48, 67-113.

Rowaihy, H., S. Eswaran, M. Johnson, D. Verma, A. Bar-Noy, T. Brown, and T. L. Porta, 2007: A survey of sensor selection schemes in wireless sensor networks. In: Proceedings of SPIE 6562, Unattended Ground, Sea, and Air Sensor Technologies and Applications.

Sahni, S., and T. Gonzalez, 1976: P-complete approximation problems. $J$ ACM, 23, 555-565.

Schlegel, M., K. Stockmann, T. Binder, and W. Marquardt, 2005: Dynamic optimization using adaptive control vector parameterization. Comput Chem Eng, 29, 1731-1751.

Shmoys, D. B., and É. Tardos, 1993: An approximation algorithm for the generalized assignment problem. Math Program, 62, 461-474.

Streeter, M., and D. Golovin, 2008: An online algorithm for maximizing submodular functions. In: Proceedings of Advances in Neural Information Processing Systems 21, 1577-1584.

Sun, X., C. G. Cassandras, and X. Meng, 2017: A submodularity-based approach for multi-agent optimal coverage problem. In: Proceedings of the 56th IEEE Conference on Control and Decision, 4082-4087.

Sviridenko, M., 2004: A note on maximizing a submodular set function subject to a knapsack constraint. Oper Res Lett, 32 (1), 41 - 43.

Tschiatschek, S., A. Singla, and A. Krause, 2017: Selecting sequences of items via submodular maximization. In: Proceedings of the 31st AAAI Conference on Artificial Intelligence, 2667-2673.

Vetta, A., 2002: Nash equilibrium in competitive societies, with applications to facility location, traffic routing and auctions. In: Proceedings of the $43 \mathrm{rd}$ Annual IEEE Symposium on Foundations of Computer Science, 416-425.

Vondrák, J., 2008: Optimal approximation for the submodular welfare problem in the value oracle model. In: Proceedings of the 40th Annual ACM Symposium on Theory of Computing, 67-74.

Wang, Z., B. Moran, X. Wang, and Q. Pan, 2014: Approximation for maximizing monotone non-decreasing set functions with a greedy method. J Comb Optim, 31, 29-43. 
Wolsey, L. A., 1982: Maximising real-valued submodular functions: primal and dual heuristics for location problems. Math Oper Res, 7, 410-425.

Zhang, Z., E. K. P. Chong, A. Pezeshki, and W. Moran, 2016: String submodular functions with curvature constraints. IEEE Trans Autom Control, 61, $601-616$. 